\title{
Single-nucleus RNA-seq identifies Huntington disease astrocyte states
}

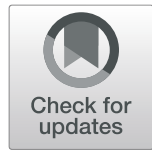

\author{
Osama Al-Dalahmah', Alexander A. Sosunov², A. Shaik³, Kenneth Ofori', Yang Liu', Jean Paul Vonsattel', \\ Istvan Adorjan ${ }^{4}$, Vilas Menon ${ }^{5}$ and James E. Goldman ${ }^{1 *}$ (I)
}

\begin{abstract}
Huntington Disease (HD) is an inherited movement disorder caused by expanded CAG repeats in the Huntingtin gene. We have used single nucleus RNASeq (snRNASeq) to uncover cellular phenotypes that change in the disease, investigating single cell gene expression in cingulate cortex of patients with HD and comparing the gene expression to that of patients with no neurological disease. In this study, we focused on astrocytes, although we found significant gene expression differences in neurons, oligodendrocytes, and microglia as well. In particular, the gene expression profiles of astrocytes in HD showed multiple signatures, varying in phenotype from cells that had markedly upregulated metallothionein and heat shock genes, but had not completely lost the expression of genes associated with normal protoplasmic astrocytes, to astrocytes that had substantially upregulated glial fibrillary acidic protein (GFAP) and had lost expression of many normal protoplasmic astrocyte genes as well as metallothionein genes. When compared to astrocytes in control samples, astrocyte signatures in HD also showed downregulated expression of a number of genes, including several associated with protoplasmic astrocyte function and lipid synthesis. Thus, HD astrocytes appeared in variable transcriptional phenotypes, and could be divided into several different "states", defined by patterns of gene expression. Ultimately, this study begins to fill the knowledge gap of single cell gene expression in $\mathrm{HD}$ and provide a more detailed understanding of the variation in changes in gene expression during astrocyte "reactions" to the disease.
\end{abstract}

Keywords: Huntington disease, Astrocytes, Gene expression, Cingulate cortex, Single-cell RNA-sequencing

\section{Introduction}

Huntington Disease (HD), a neurodegenerative disorder caused by CAG repeats in the Huntingtin gene, leads to the accumulation of the mutant protein and an associated neuronal degeneration and gliosis [34]. Although Huntingtin is expressed in all cell types, the neuropathology of the disease shows substantial variation. For instance, there are caudal-rostral and medial-lateral gradients of severity in the neostriatum [57], and relative sparing of the nucleus accumbens in advanced grade HD [44]. Neuronal degeneration in the isocortex involves the motor and sensory cortices but relatively spares the superior parietal lobule [37], and in some patients, the anterior cingulate cortex is also involved, and cortical layers III, V, and VI are especially vulnerable [45].

\footnotetext{
* Correspondence: jeg5@cumc.columbia.edu

${ }^{1}$ Department of Pathology \& Cell Biology, Columbia University, New York City, NY, USA

Full list of author information is available at the end of the article
}

Understanding the differences in microenvironment between affected and relatively resistant regions may illuminate the cellular and molecular mechanisms underlying vulnerability and resilience to neurodegeneration. Astrocytes, in particular, are a key to maintaining the neuronal microenvironment, and display regional variation across the dorsal-ventral, rostral-caudal, and medial-lateral axes according to developmentally-determined domains [55]. Additionally, astrocytes exhibit intra-regional heterogeneity. For example, subpial and white matter astrocytes contain more glial fibrillary acidic protein (GFAP) and CD44 than protoplasmic astrocytes but lower levels of protoplasmic astrocyte markers, such as glutamate transporters and glutamine synthetase [51]. Astrocytes in the affected regions of the HD brain show a "reactive" state, generally defined by histochemistry or by an increase in GFAP $[48,58]$, but also by a decrease in the expression and protein levels of the major astrocytic glutamate transporter, EAAT2 $[2,9]$. In mouse models of HD, expression 
of mutant Huntingtin (mHTT) in astrocytes leads to decrease in the expression of the glutamate transporter [5], and the failure to buffer extracellular potassium and glutamate leads to neuronal hyper-excitability and a HD-like phenotype, which is reversed by restoration of astrocytic membrane conductance [53]. In addition, astrocytes in HD contribute to an inflammatory environment [16, 27], which likely participates in the progression of the pathology. Thus, there is substantial evidence that astrocytes play a primary role in the evolution of HD.

Whereas bulk transcriptome-wide studies have provided important insights into molecular changes in HD $[1,14,15,23,29,38]$, bulk samples comprise a mixture of cell types, so astrocyte-specific signatures in HD may be obscured. Single cell RNA sequencing (scRNAseq) is a powerful technique to interrogate cellular heterogeneity [6, 42]. Although brain banks house hundreds of frozen postmortem brain specimens, technical difficulties limit the application of scRNAseq to these samples. However, this is not a limitation of single nucleus RNA sequencing (snRNASeq), which accurately elucidates cellular heterogeneity in a manner comparable to whole/ cytoplasmic scRNAseq [25], and can be applied to frozen brain tissue [22]. This technique has been used to identify multiple novel, regionally-diversified cortical excitatory and inhibitory neuronal sub-types [24]. Recently, massively parallel snRNASeq using droplet technology revealed cellular heterogeneity in the human postmortem cortex and hippocampus [11, 17]. The diseasespecific cell-type specific transcriptional signatures were described in oligodendroglia in multiple sclerosis [18] and multiple cell types in Alzheimer Disease [31] and microglia in Alzheimer disease [39].

In this study, we performed snRNASeq of fresh frozen cingulate cortex from Grade III/IV HD patients and compared the results to those from non-neurological control patients to examine single cell differences in gene expression. We have focused here on astrocytes, although we found significant differences in all cell types. We examined the cingulate cortex, which is often affected in HD patients, because the cortical pathology is less severe than the neostriatal pathology, and because there is little known about cortical astrocyte pathology in HD. Finally, we focused on Grade III/IV to identify astrocyte gene expression changes in an intermediate stage of evolution rather than at an end stage.

Overall, we found substantial heterogeneity in astrocyte signatures, with clear differences in population structure between control and HD tissue samples. In particular, the "reactive" astrocytes in HD could be divided into several different "states", defined by patterns of gene expression. These ranged from astrocytes that had markedly upregulated metallothionein (MT) and heat shock genes, but had not completely lost the expression of genes associated with protoplasmic astrocytes, to astrocytes that had substantially upregulated GFAP and had lost expression of many normal protoplasmic genes as well as the MT genes. The variation in astrocytes is not surprising, given that $\mathrm{HD}$ is a degenerative disease that progresses over years and one would not expect all astrocytes to respond synchronously during the course of the disease.

Studies like this one will begin to fill the knowledge gap of single cell gene expression in HD and give us a more detailed understanding of the variation in changes in gene expression during astrocyte "reactions." Furthermore, network building from these gene sets will help define interacting genes and important regulatory genes behind reactive astrocyte states.

\section{Methods}

Dissection of the cingulate cortex from frozen tissue

Postmortem anterior cingulate cortex specimens frozen during autopsy from control and grade III/IV HD were obtained from the New York Brain Bank. Four cases (two HD and 2 control) were selected for snRNAseq and 12 cases (6 and 6) for bulk RNAseq, all with RNA integrity numbers of $>7$. Cortical wedges measuring $\sim 5 \times$ $4 \times 3 \mathrm{~mm}$ were dissected on a dry ice cooled stage and processed immediately as described below. A table of the cases and controls used is provided in Table 1.

\section{Single nucleus RNAseq}

Nuclei were isolated as described in [22]. Briefly, cortical tissue was homogenized in a Dounce homogenizer with 10-15 strokes of the loose pestle and 10-15 strokes of the tight pestle on ice in a Triton X-100 based, sucrose containing buffer. The suspension from each sample was filtered through a BD Falcon tubes with a cell strainer caps (Becton Dickinson, cat. no. 352235), washed, refiltered, washed, followed by a cleanup step using iodixanol gradient centrifugation. The nuclear pellet was then re-suspended in 1\% BSA in nuclease-free PBS (containing RNAse inhibitors) and titrated to 1000 nuclei/ $\mu \mathrm{l}$. The nuclear suspensions were processed by the Chromium Controller (10x Genomics) using single Cell 3' Reagent Kit v2 (Chromium Single Cell 3' Library \& Gel Bead Kit v2, catalog number: 120237; Chromium Single Cell A Chip Kit, 48 runs, catalog number: 120236; 10x Genomics).

\section{Sequencing and raw data analysis}

Sequencing of the resultant libraries was done on Illumina NOVAseq 6000 platformV4 $150 \mathrm{bp}$ paired end reads. Alignment was done using the CellRanger pipeline (10X Genomics) to GRCh38.p12 (refdata-cellrangerGRCh38-1.2.0 file provided by 10x genomics). Count matrices were generated from BAM files using default parameters of the DropEst pipeline [41]. Filtering and 
Table $1 \mathrm{HD}$ and Control Patient Data

\begin{tabular}{|c|c|c|c|c|c|c|c|c|}
\hline Patient & Age & Gender & $\begin{array}{l}\text { CAG } \\
\text { Repeats }\end{array}$ & Other Neuropathology & Cause of Death & RNASeq & PMI ${ }^{\mathrm{COLD}}$ & PMIFROZEN \\
\hline HD5493 & 48 & $\mathrm{~F}$ & $46-25$ & Glial scars, dentate, cerebellum, small & PE, pneumonia & $S N+B U L K$ & N/A & $9: 33$ \\
\hline HD5575 & 71 & M & $44-18$ & AA, $\mathrm{H} / \mathrm{I}$ acute & Cardiac Arrest & $\mathrm{SN}+\mathrm{BULK}$ & $0: 07$ & 15:04 \\
\hline HD5301 & 54 & M & $46-19$ & AA, $\mathrm{H} / \mathrm{I}$ acute & N/A & BULK & $1: 27$ & 18:52 \\
\hline HD4929 & 50 & M & $49-16$ & $\mathrm{H} / \mathrm{l}$ acute & Inanition & BULK & 0:05 & 43:08 \\
\hline HD3859 & 36 & M & $50-17$ & NS & N/A & BULK & $0: 40$ & $15: 00$ \\
\hline HD5300 & 53 & $\mathrm{~F}$ & $46-22$ & NS & Pneumonia & BULK & $0: 58$ & 20:03 \\
\hline C5382 & 62 & M & & AA & Acute,PE & $S N+B U L K$ & N/A & $5: 24$ \\
\hline C5404 & 54 & $\mathrm{~F}$ & & AA & Acute, Ml & $S N+B U L K$ & $6: 41$ & $16: 36$ \\
\hline C4812 & 36 & M & & $\mathrm{H} / \mathrm{I}$ acute & Acute, Ml & BULK & N/A & $8: 37$ \\
\hline C180 & 52 & $\mathrm{~F}$ & & AA, H/l acute, infarcts, $C$, precentral gyrus & N/A & BULK & $2: 25$ & $6: 07$ \\
\hline C98 & 55 & M & & AA & Acute collapse & BULK & 6:05 & $37: 00$ \\
\hline C4518 & 56 & M & & AA, focal, old hem \& infarcts, thalamus, stem, basal ganglia & Acute cardiac arrest & BULK & N/A & 8:50 \\
\hline
\end{tabular}

PE Pulmonary embolism, MI Myocardial infarction, AA Athero-arteriosclerosis, NS No other significant neuropathology, $H / I$ Hypoxic/ischemic changes, $C$ Caudate, $N$ / $A$ Not available. PMI ${ }^{\mathrm{COLD}}$ hours between death and preservation of brain on ice. PMI ${ }^{\mathrm{FROZEN}}$ hours between preservation on ice and freezing. Note that some of these patients died outside of Columbia University Medical Center and brains were sent here on ice. Cause of death was not available for some patients who died outside the medical center and whose autopsy was limited to the brain

QC was done using the scater package [32]. Nuclei with percent exonic reads from all reads in the range of $25-$ $75 \%$ were included. Nuclei with percent mitochondrial reads aligning to mitochondria genes of more than $14 \%$ were excluded. Genes were filtered by keeping features with $>10$ counts per row in at least in 31 cells.

\section{Normalization and data-cleanup}

The count matrix was normalized by first running the quickcluster function, then estimating sizefactors by calling scran::computeSumFactors() function with default options and clusters set to clusters identified by calling quickcluster function. Scater::normalize function was then used to generated normalized counts. Of note, batch effects were taken into account when normalizing the data (Scater package in R). Doublet identification was done using scran::doubletCells function with default options, and cells with doublet score of $>=1.5$ were excluded. A total of 5199 cells passed QC at this point (C5382: 893 nuclei, H5575: 646 nuclei (Batch1), and C 5404: 1096 nuclei, H5493: 2151 nuclei (Batch 2)). We filtered low-quality nuclei using the percentage of the celllineage specific genes that are expressed (Identity score - see below). If the cell's identity score was less than $20 \%$, a nucleus was considered low-quality and was excluded. A total of 4786 nuclei remained after this step.

\section{Clustering and classification of nuclei}

Clustering, cell-lineage assignment, and sub-clustering was done as follows. First, an unsupervised pre-clustering step was performed to split the nuclei into small preclusters. Second, the pre-clusters were assigned a class using gene set enrichment analysis and GO term enrichment analysis of the pre-cluster markers. Third, mixed pre-clusters (with mixed enrichment scores for lineage genes) were identified, and the nuclei within these clusters were assigned to new pre-clusters based on their highest identity score (see below). Fourth, the pre-clusters were agglomerated into master classes (Astrocytes, Neurons, Microglia, Endothelial cells, Oligodendrocytes, and Oligodendrocyte Precursor Cells (OPCs). Finally, master classes were sub-clustered using the SC3 clustering algorithm. These steps are described in Additional file 1: Figure $\mathrm{S} 7$ and in detail below.

\section{Pre-clustering}

First, dimensionality reduction methods including tSNE and PCA (prcomp) were conducted using functions provided in scater package. Briefly, dimensionality reduction by tSNE was done using scater::runtSNE() or runPCA() functions. For runPCA () function, a random seed was set (12345) and theta was set to default (0.5). Multiple perplexity levels were tested -a level of 527 was empirically chosen. We then used an unbiased clustering approach using shared nearest neighbor utilizing the scran::buildSNNGraph() function, with dimensionality reduction set to "TSNE". Multiple $\mathrm{K}$ values were tested (6-16) and the number of pre-clusters generated was examined. $K=6$ yielded 35 discrete pre-clusters, with the least number of mixed pre-clusters (based on results from the cell classifier- below). $K=6$ was thus chosen for further clustering. We appreciate that using a highly nonlinear reduction such as $\mathrm{tSNE}$ as input for building a SNN graph is not preferable to using PCA. However, our experience is that using tSNE reduced dimensions yielded fewer pre-clusters with mixed signatures of well- 
studied, known cell classes. Moreover, we used an independent method to ascertain the quality of the preclusters (see next section).

We developed a simple algorithm to assign cell classes based on the highest proportion of cell-class specific genes (referred to thereafter as Cell classifier tool). The tool also classifies nuclei into master classes (Neurons, Astrocytes, Oligodendrocytes, OPCs, Microglia, and Endothelial Cells). More specifically, each nucleus is given an identity score that is equal to the percentage of genes that are expressed from a cell-class specific gene list. The gene lists used to classify nuclei are based on the literature $[36,63]$, modified, and are provided (Additional file 2: Table S1). Each nucleus is given identity scores for all 6 cell types: Neuron, Astrocyte, Oligodendrocyte, OPC, Microglia, and Endothelial. The cell is assigned the class (identity) for which it scores the highest identity score. Additionally, an ambiguity status is assigned based on whether the curve of identity scores is skewed (Unambiguous) towards the highest score versus flattened (Ambiguous). More specifically, if the sum of second and third highest identity scores is higher than $2 \mathrm{X}$ the highest identity score, the identity curve is flattened, and the cell is considered ambiguous. This helps identify problematic nuclei in mixed clusters as explained in the section below.

\section{Pre-cluster identity assignment}

To assign pre-cluster identities, we used multiple approaches to ascertain pre-cluster and nuclei identities. First, we used the mean of normalized counts per gene per cluster to perform gene set enrichment analysis using the gsva R package ('gsva' method with $\mathrm{kcdf}=$ "Gausian" and $m x$.diff $=$ FALSE). We used cell-type specific genesets based on a literature search (provided in Additional file 2: Table S1 - the same as those used for the cell classifier tool). Second, we manually examined the top marker genes that differentiate pre-clusters and determined whether any pre-clusters showed lineage-discordant marker genes. For example, if one pre-cluster was characterized by oligodendrocytic (MBP, TMEM120, MOBP) as well as neuronal (CCK, MAP1B, BEX3, NRGN) or microglial (HLA-B, IBA1, C1QB) genes, a cluster was considered mixed. Finally, we also performed GO term enrichment analysis on the top marker genes and manually examined the terms that distinguished each cluster. Pre-cluster markers were discovered using the scran::findmarkers() function with default options.

The majority of pre-clusters were homogeneous, and showed high enrichment scores in one lineage. However, some pre-clusters, like pre-clusters $2,7,27$, and 17, were mixed, showing high enrichment scores for microglial and oligodendrocytic genes (2 and 17), or astrocytic and neuronal genes (7 and 27). Thus, we used supervised classification, as above, and re-assignment of the nuclei in the ambiguous clusters based on the maximum score assigned by the cell classifier scores (cluster_max_score). This resulted in the following pre-clusters: Microglia_r2_17, Oligodendrocyte_r2 17, Astrocyte_r2_17, Neuron_r17_2, Endothelial_r2, Astrocyte_r7_1, Astrocyte_r27_2, Neuron_r7_1, and Neuron_r27_ 2 . In addition, cluster 31 showed mixed neuronal and microglial enrichment scores, but based on the cell classifier scores the majority of cells $51 / 59$ were designated as neurons, and only one cell was called microglial. (Astrocyte- 6, endothelial cells- 0, Microglial-1, Neuron-51, Oligodendrocyte -1). Thus, this cluster was renamed as 31_Neuron. Note, for endothelial cells, only supervised assignment was able to detect a discrete cluster.

Afterwards, clusters of the same lineage were conglomerated into master classes: Astrocytes (1064 nuclei), Endothelial cells (19), Microglia (147), Neuron (2085 nuclei), Oligodendrocytes (1230 nuclei), and OPC's (241 nuclei).

For neuronal clusters, a minority of nuclei expressed astrocytic genes despite the neuronal identity being the highest as assigned based on the cell-classifier results. These cells had ambiguous scores per the cell classifier. Thus, all neuronal nuclei that were classified as ambiguous were excluded (219 nuclei), leaving 1866 nuclei for downstream analysis (to do SC3). GFAP and AQP4 genes were excluded from the analysis of neuronal nuclei (the counts were re-normalized without reads from GFAP/AQP4 genes).

\section{Consensus clustering using $\mathrm{SC} 3$ for sub-clustering}

To determine objectively the optimal way to cluster the astrocytic, oligodendrocytic, and neuronal nuclei, we used consensus clustering as described in the SC3 package [19]. Values for K (number of clusters in K-mean clustering) were empirically tested and silhouette widths average values for the resultant clusters were examined. For astrocytes $K=6$ was chosen as silhouette values were all above 0.5 (except cluster 2, an HD astrocytic cluster with both GFAP expression and MT gene expression. We decided to keep this cluster as it made biological sense. Cluster markers (generated through the SC3 pipeline) were then generated using pairwise $t$-test. To find marker genes (SC3 package), a binary classifier was constructed as informed by the mean cluster expression values for each gene. Prediction accuracy was tested using the area under the receiver operating characteristic (AUROC) curve. Wilcoxon signed rank test $p$-values were assigned per gene. Here we used (AUROC) $>0.65$ and with the $\mathrm{p}$-value $<0.05$. Cluster markers were also generated using pair-wise t-test (as per scran library in R). Note, for the astrocytic sub-cluster gene list used to classify astrocytic nuclei, the following options were using for the scran::findmarkers() function: log fold change 0.5 and markers were detected in "any" direction. A selected list of astrocytic sub-cluster top gene markers 
and cell-type cluster markers are provided in Table 2 and Additional file 2: Table S1, respectively. These lists were generated as follows: For each cluster, the log-fold change values for each gene were summed across the other clusters. The genes were then ranked in descending order by these sums. Genes with one or more negative $\log$-fold changes or FDR-corrected $p$ values $\geq 0.05$ were excluded from the marker list, even if the sum of the log-fold changes was high. This imparts specificity to the markers selected for each cluster.

\section{Supervised classification of astrocytes}

To classify astrocytic nuclei into astrocytic states, we used monocle 2.0 [54]. We used the following rules to assign astrocytes into four states based on logtransformed expression values: Quiescent- SLC1A2 > = 2, MT2A < 4, and GFAP <3; State-1Q: SLC1A2 > = 2, $\mathrm{MT} 2 \mathrm{~A}>=4$, and GFAP $<3$; State-2R: $\mathrm{SLC} 1 \mathrm{~A} 2<2$, MT2A $>=4$, and GFAP $>=3$; and State-3R: SLC1A2 $<2$, $\mathrm{MT} 2 \mathrm{~A}<4$, and GFAP $>=3$. Events that did not meet any of the conditions, or met more than one condition were classified as unknown or ambiguous, respectively.

Differential gene correlation analysis and gene multiscale embedded gene co-expression network analysis

Differential gene correlation analysis and gene network analysis were done in DGCA [35] and MEGENA [49] R packages, respectively. In brief, the count matrix was

Table 2 Astrocyte sub-cluster markers

\begin{tabular}{|c|c|c|c|c|c|c|}
\hline Cluster_1 & Cluster_2 & Cluster_3 & & Cluster_4 & Cluster_5 & Cluster_6 \\
\hline MT2A & HSP90AA1 & COL5A3 & MICALL2 & APOE & MALAT1 & ID2 \\
\hline MT1F & HSPB1 & FGFR3 & ATP1B2 & PTN & GFAP & $\mathrm{S} 100 \mathrm{~B}$ \\
\hline MT1E & CRYAB & C1orf61 & CARMIL1 & TTYH1 & NEAT1 & FOS \\
\hline MT1X & MTATP6P1 & NTM & CAPN2 & GLUL & FP236383.2 & $\mathrm{DBI}$ \\
\hline MT1G & HSPA1A & AC007325.2 & NPIPB12 & CALM2 & SORBS1 & HES1 \\
\hline MT1M & UBC & SLC1A2 & ATP1A2 & SLC1A3 & PLP1 & JUNB \\
\hline FTL & CALM1 & CIRBP & ACSL6 & WIF1 & CEBPD & FABP7 \\
\hline MT3 & HSP90AB1 & ADGRV1 & SYNE1 & SLITRK2 & TSC22D1 & H3F3B \\
\hline FAM171B & ATP1B1 & NAT8L & ADGRG1 & TNIK & PRRG3 & ID3 \\
\hline TPD52L1 & GPM6B & NCAN & COL16A1 & AC092535.1 & SP100 & PTGDS \\
\hline EGLN3 & CCK & SFXN5 & AL391807.1 & MAP1B & HILPDA & MT1H \\
\hline CPE & VAMP2 & SON & ACSS1 & SDC4 & COL6A3 & IFITM3 \\
\hline GJB6 & MAP1A & SNRNP70 & GABBR1 & TUBB2A & RASD1 & \\
\hline EPHX1 & NRGN & MIAT & LINC00982 & CKB & CHI3L1 & \\
\hline TKT & EEF1A1 & LCNL1 & ADGRB1 & HNRNPL & SLC14A1 & \\
\hline CLU & EIF1 & NDRG2 & ANKDD1A & CXCL14 & SAT1 & \\
\hline RGCC & TMSB4X & SRRM2 & LSAMP & F3 & HNRNPH1 & \\
\hline \multirow[t]{14}{*}{ NUPR1 } & & LUC7L3 & ACADVL & HES4 & PCDH9 & \\
\hline & & DST & RNA5-8S5 & TMSB10 & PRKCA & \\
\hline & & MFGE8 & RNA5-8SN5 & PON2 & BCL6 & \\
\hline & & MTSS1L & LGI4 & BEX1 & LINC00969 & \\
\hline & & MACF1 & FNBP1 & ITM2C & ANKRD36B & \\
\hline & & IQCA1 & GOLGA8B & SNAP25 & HIF3A & \\
\hline & & NPIPB5 & & PCSK1N & ITGB8 & \\
\hline & & PLXNB1 & & RNA5-8SN1 & DTNA & \\
\hline & & NPIPB3 & & IGFBP7 & HSPA1B & \\
\hline & & PDGFRB & & DDX17 & ETNPPL & \\
\hline & & & & RBM6 & & \\
\hline & & & & GAN & & \\
\hline & & & & TOB1 & & \\
\hline & & & & ACTB & & \\
\hline
\end{tabular}


filtered to keep the top 5\% most highly expressed astrocytic genes by average using the filtergenes() function with the following options: filterTypes = "mean" and filterCentralPercentile $=0.95$. The resultant matrix had a total of 856 genes. The DGCA pipeline was performed using the ddcorAll() function with the following options: adjust = " perm", nPerm $=100, n$ nPairs $=1000$. Visualization was done in ggplot2 [61]. Identification of astrocytic gene modules was done in MEGENA $R$ package with default options ( $\min$ size $=10$ ). GO term enrichment analysis of genes in the modules was done using the moduleGO() function in DGCA R package with a $p$ value set at 0.05 . Gene set variation analysis of module genes was done using the GSVA() R package as described below.

\section{Gene set variation analysis (GSVA)}

The average normalized count per gene per cluster was calculated. The resultant cluster-wise count matrix was used as input to the GSVA pipeline [12]. Gene sets used for various tests are provided in the Additional file 2: Table S1. The options used for performing the GSVA pipeline are as follows: method = 'gsva', $k c d f=$ "Gaussian", mx.diff = FALSE. Heat maps were generated using the heatmap. 2 in $\mathrm{R}$ function from the package gplots $[60]$ and scores z-scaled were indicated.

\section{Bulk RNAseq}

RNA was extracted from cingulate cortical specimens using Trizol ${ }^{\mathrm{Tm}}$ method. RIN values were determined, all samples had values>7.0. Sequencing was done on Illumina Hiseq $2000^{\mathrm{m}}$ platform. Raw reads were aligned to the reference (GRCh38.p12) using STAR aligned [8]. Ribosomal RNA reads were removed using SortMeRNA [21]. For data analysis was done in R v 3.5 in Linux or Windows 10 environment. Genes with fewer than 10 reads per row were excluded. Principal component analysis of normalized variance stabilized counts (product of DESeq2::varianceStabilizingTransformation) in FactoMineR $\mathrm{R}$ package [26] revealed that the first two components explained $47.4 \%$ of the variance in the data, and that the variance was captured entirely in the first 11 components. Condition (HD versus control) was the variable that is best correlated with $\mathrm{PC} 1$ (eta2 $=0.62$, $\cos 2=0.88$ ). After variance stabilization, library size was not correlated with PC1 (correlation coefficient 0.08). For differential gene expression and statistical analysis, EdgeR [33, 43] with incorporating age and gender into the design matrix. The likelihood ratio test (LRT) method was used with an adjusted $p$ value of 0.05 and an absolute log fold change threshold set at 1.4. Principal component analysis and visualization were done using DESeq2 package [30]. Sample distances were calculated from normalized counts (DESeq2) using the Manhattan metric using the $\operatorname{dist}()$ function from base package in $\mathrm{R}$ (v3.5.2). pheatmap from pheatmap package was used for visualizing utilizing the ward method for clustering [20]. Gene Ontology term analysis was done in gProfiler web platform (https://biit.cs.ut.ee/gprofiler/ gost) by supplying an ordered query and determine statistical significance using the Benjamini-Hochberg FDR method $(p<0.05)$. The terms shown in the Figures are selected based on ordering the results based on negative_log10_of_adjusted_p_value followed by the ratio of the shared of number of genes enriched in a term to that of the total number of genes in the GO term (desc(intersection_size/term_size)).

\section{Quantitative PCR}

Total RNA was extracted from brain specimens using TRIzol reagent (Invitrogen, Carlsbad, CA, USA). RNA concentration and purity were determined using NanoDrop (Thermo Scientific ${ }^{\mathrm{T \omega}}$, MA). A bioanalyzer was used to determine RNA integrity. RNA was converted to cDNA using High capacity RNA-to-cDNATM kit (Thermo Fisher Scientific, Applied BiosystemsTM,MA). Specific qPCR primers were designed using Primer3 and from Primer Bank. Each $15 \mu \mathrm{L}$ qPCR reaction contained $5 \mathrm{ng}$ of cDNA, 7.5 $\mu \mathrm{L}$ of 2- SsoAdvanced ${ }^{\mathrm{m}}$ Universal SYBR ${ }^{\circ}$ Green Supermix (Biorad, PA), $400 \mathrm{nM}$ of each primer and nuclease free water. The $\mathrm{qPCR}$ plates were read on a Mastercycler ${ }^{\circ}$ RealPlex2 (Eppendorf, NY). The reactions were done in triplicates. Relative gene expression was calculated using the delta delta Ct Pfaffl method with UBE2D2 and RPL13 as reference genes (Pfaffl MW. 2001). Statistical analysis was done using one-tailed Mann Whitney $U$ test. The GFAP primers were AGGTCC ATGTGGAGCTTGAC (forward) and GCCATTGCCT CATACTGCGT (reverse) and $A C T N B$ primers were CTGGAACGGTGAAGGTGACA (forward) and AAGG GACTTCCTGTAACAATGCA (reverse) [62].

\section{Human brain tissue processing, histology, in situ hybridizations, and immunohistochemistry}

Standard H\&E and Cresyl violet histochemical stains were done in the histology core at the Department of Pathology and Cell Biology at Columbia University. The cases and controls used are provided in Table 1. Standard chromogenic and fluorescent immunohistochemistry was done as described previously in Sosunov A et al. [51] in paraffin-embedded formalin-fixed tissue sections or fresh frozen sections briefly fixed in 4\% PFA, for 10 min $\left(4^{\circ} \mathrm{C}\right)$ in $4 \%$ PFA in PBS. Paraffin sections after deparaffinization were treated with Antigen Unmasking Solution according to the manufacturer's recommendations (Vector Laboratories, Burlingame, CA). GFAP and Huntingtin dual immunohistochemical (IHC) stains were done using standard DAB and alkaline phosphatase dual staining on a Leica Bond ${ }^{\mathrm{Tm}}$ auto-stainer. The 
following antibodies and dilutions were used GFAP (rabbit polyclonal, 1:1000, DAKO Z0034 or chicken polyclonal, Abcam Cat\# ab4674, 1:300), CD44 (rat monoclonal, 1:100, Millipore A020), glutamine synthetase (GS) (mouse monoclonal, 1:1000, Transduction Laboratories 610,518), C3 (rabbit monoclonal, 1:200, Abcam Ab20999), metallothionein (MT) (mouse monoclonal, 1:200, Abcam ab12228), HTT (mouse monoclonal, 1:2000 Millipore MAB5492 1:2000), ALDH1L1 (mouse monoclonal, Encor Cat\# MCA-2E7, 1:100), IBA1 (Wako \# 019-19,741 Rabbit polyclonal, 1:300-1:500), LN3 (mouse monoclonal, 1;75, MP Biomedicals LLC, \#69303). For fluorescent IHC, secondary antibody conjugated to fluorophores: anti-mouse Alexa Fluor 488 and 594, anti-rabbit Alexa Fluor 488 and 594, and anti-chicken Alexa Fluor 620; all from goat or donkey (1:300, ThermoFisher Scientific, Eugene, OR) were applied for $1 \mathrm{~h} \mathrm{RT}$. In situ hybridization was done using RNAscope $^{\mathrm{Tx}}$ multiplex fluorescent v2 (ACDbio cat no 323100 ) per the manufacturer's protocol in 5- $\mu \mathrm{m}$ paraffinembedded, formalin-fixed tissue sections. We used custom designed probes for GFAP and PLP-1 (cat no.'s 584, 791-C3 and 564,571-C2, for GFAP and PLP-1, respectively). The probes were designed to cover all transcripts. We used an available probe for MBP (Cat. no. 573051). Images were taken on a Zeiss 810 Axio confocal microscope. Brightfield and chromogenic images were taken on an Aperio LSM ${ }^{\mathrm{mt}}$ slide scanner at 20X (Brightfield).

\section{Results}

\section{Major gene expression alterations in the HD cingulate} cortex

To explore gene expression alterations in the cingulate cortex, we analyzed bulk RNA expression profiles from six grade III and grade IV HD and six non-neurologic controls (Table 1). Analysis of sample distance showed HD samples clustered together, except for one case of Juvenile onset HD (H3859) (Fig. 1a). A recent report suggests that in juvenile HD the cerebral cortex is largely unchanged compared with controls [52]. We next performed differential gene expression analysis from bulk RNAseq specimens, and identified 3165 downregulated genes and 1835 upregulated genes at a BenjaminiHochberg adjusted false discovery rate of 0.05 (Fig. 1b). Reactome pathways and GO terms enriched in the upregulated genes revealed an enrichment in HD of multiple immune response genes including complement, toll-like receptor signaling, and Interleukin pathways, featuring IL-13 and IL-10 (Fig. 1d). In addition, genes involved in responses to metal ions, metal sequestration, and metallothionein binding were also enriched in HD. GO term analysis of genes reduced in HD revealed that the majority of these genes were associated with neuronal identity or function (Additional file 3: Figure S5D). This is further discussed in the Additional file 4: Results.
A full list of the top differentially upregulated and downregulated genes in HD is provided in Additional file 5: Table S5.

Next, we examined astrocytic genes that were differentially expressed in HD. We chose to look at the expression of astrocyte genes from Zamanian et al. and Liddelow et al. [27, 63], described as A1 (neurotoxic), A2 (neuroprotective), and pan-reactive astrocytic genes (Fig. 1c). We found that a subset of A1, A2, and panastrocytic genes were significantly increased in $\mathrm{HD}$, including GFAP, CD44, OSMR, FKBP5, STEAP4, and CXCL10 for pan-reactive genes, C3, SRGN, and GBP2 for A1 genes, and EMP1, CD14, and CD109 for A2 genes. In contrast, the gene $A M I G O 2$, an A1-specific gene, was reduced in HD. We next performed geneset enrichment analysis of select astrocytic genesets (astrocyte differentiation and astrocyte markers [28]) and found them enriched in HD (Fig. 1e-f). Of note, there was significant heterogeneity in differential gene expression of astrocytic genes among HD cases. For example, RNA expression profiles from the aforementioned juvenile HD case (H3859) as well as another grade IV HD case (H4929) showed little upregulation of astrocytic genes (Fig. 1C). We investigated this further using immunohistochemistry for astrocytic markers GFAP, GS, and ALDH1-L1 (Additional file 6: Figure S1G). The results showed no qualitative changes in pattern of staining or densities of astrocytes in the cingulate cortex in case H3859 compared to non-neurologic controls (Additional file 6: Figure S1G). Taken together, the data show major gene expression changes in the HD cingulate cortex, involving upregulation of immune genes as well as astrocytic genes.

\section{Single nucleus RNAseq from control and HD cingulate cortices identifies major cell types}

The results of the bulk RNAseq showed heterogeneity within the HD cases, with some cases not showing significant differences in expression of astrocytic genes compared to controls (T4929 and T3859). Moreover, a subset of A1 "neurotoxic", A2 "neuroprotective", and pan-reactive genes were increased HD, and it was not clear whether this represents a coexistent increase in A1 and A2 astrocytes, versus increased expression of A1 and A2 genes in the same cells. To address the issue of astrocytic heterogeneity, we selected 2 HD (grade III) and 2 control cases, extracted nuclei from the cingulate gyrus, and then performed snRNAseq using the 10X Genomics Chromium $^{\text {Tw }}$ droplet-based single cell RNAseq platform (Fig. 2a). Four thousand seven hundred eightysix nuclei passed quality control measures, of which 1989 were control and 2797 were HD nuclei, as shown in the $\mathrm{t}$-distributed stochastic neighbor embedding (tSNE) plot in Fig. 2b. We used both unsupervised 


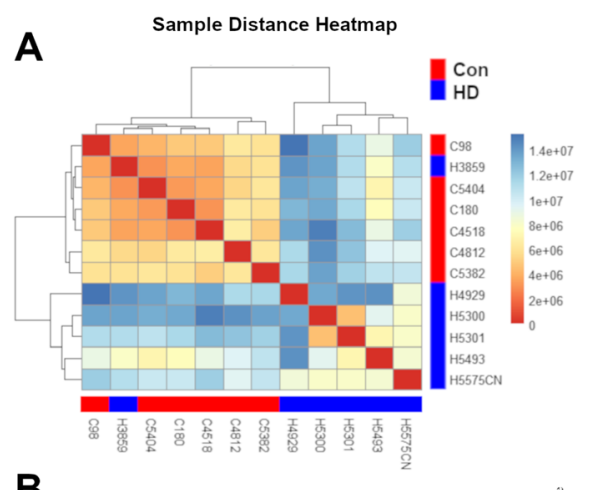

B

D
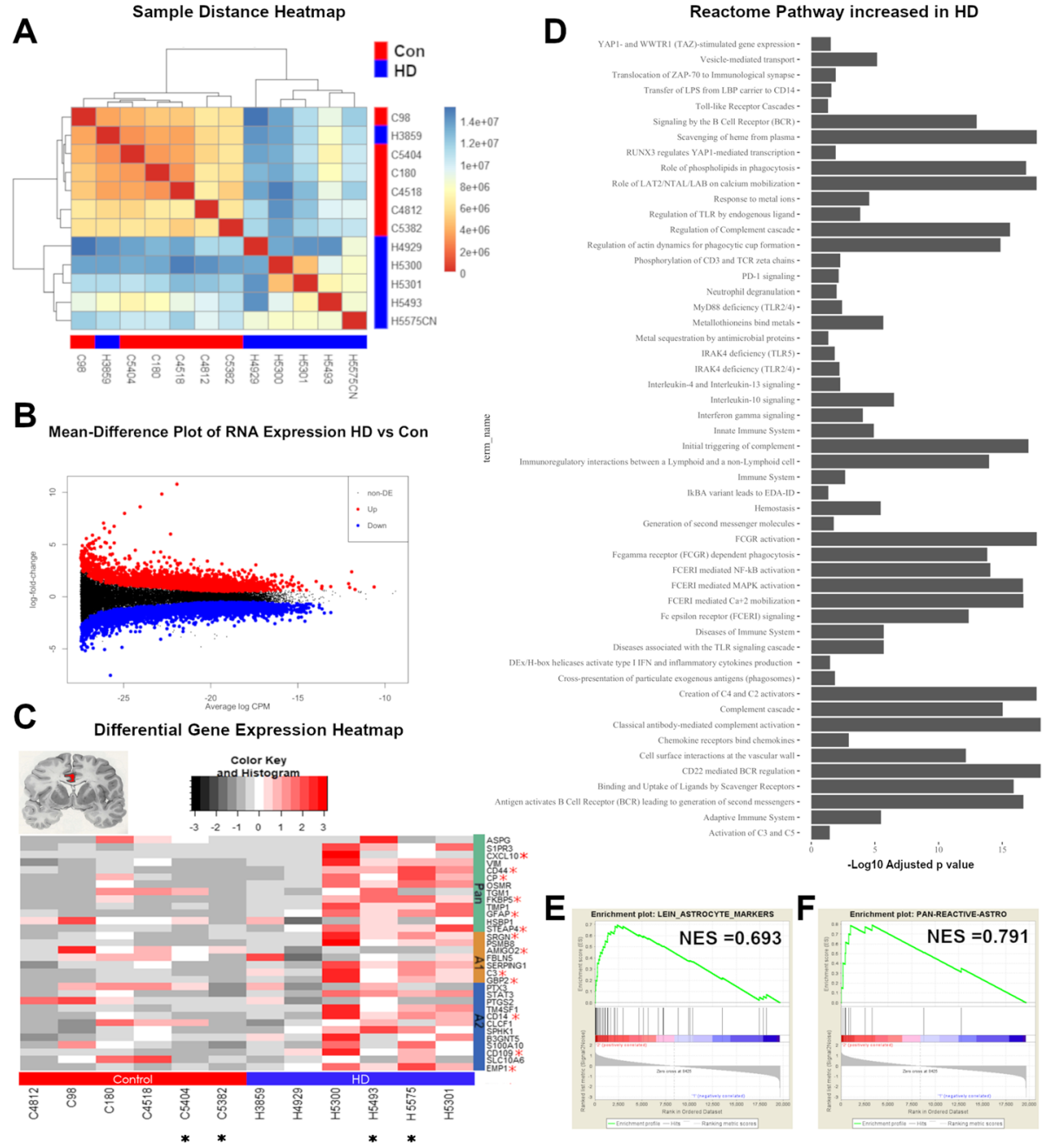

E
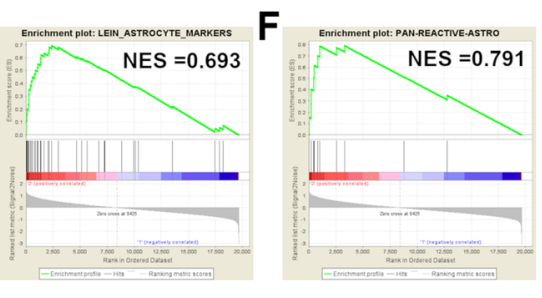

Fig. 1 Bulk_RNASEQ. Transcriptomic analysis of Huntington disease cingulate cortex. Total RNA sequencing was done on 6 grade III/IV and 6 control cingulate cortices. a Sample distance (Manhattan method) heatmap clustered using the Ward method. b Mean-Expression plot showing log2 foldchange (LFC -HD versus control) on the $y$-axis, and mean normalized counts on the $x$-axis. Significantly differentially expressed genes are shown in red and blue for upregulated and downregulated genes, respectively. c Differential gene expression heatmap of select astrocytic genes as described in Liddelow et al. [27], with controls (Con) denoted by the red bar, and HD by the blue. Asterisks next to gene names indicate significance (BenjaminiHochberg adjusted $p$ value $<0.05$ and absolute log fold change $>=1.5$ ). A1, A2, and pan-reactive astrocytic genes are denoted. Asterisks below case numbers indicate which cases were selected for single cell nuclear RNAseq. $\mathbf{d}$ Representative GO ontology term analysis showing significantly increased Reactome pathways in HD cases from bulk RNAseq (using the gProfiler web-platform, Benjamini-Hochberg adjusted $p$-values set at $<0.05$ ). e-f Gene set enrichment analysis of select astrocytic genes (Astrocyte markers and Astrocyte_differentiation -GO0048708). Normalized enrichment scores (NES) are shown

clustering and supervised classification to identify cell types, including neurons, astrocytes, oligodendrocytes, OPCs, microglia, and endothelial cells (Fig. 2c). Scaled normalized gene expression in individual nuclei per celltype displayed in a heatmap show distinct gene expression profiles among nuclei in different cell types (Fig. $2 d)$. Gene-set variation analysis of the average normalized gene expression per cell type against cell-type specific gene-sets derived from thorough assessment of the literature and genes from Gill B. et al. [10] (Additional file 2: Table S1A) show high enrichment of cell-type specific gene-sets in the classified cell types (Additional file 7: Figure S2A-B). The proportions of each cell type per condition are shown in Additional file 7: Figure S2C. While the proportions of astrocytes (24\% controls and $21 \% \mathrm{HD}$ ) and microglia (3\% each) were comparable, the proportions of neurons (53\% controls and 37\% HD) and oligodendrocytes (15\% controls and 33\% HD) were divergent. We cannot ascertain whether this is due to technical versus biological effects. The numbers and proportions of nuclei per cell type per case are shown in Additional file 7: Figure S2D-E, respectively. 


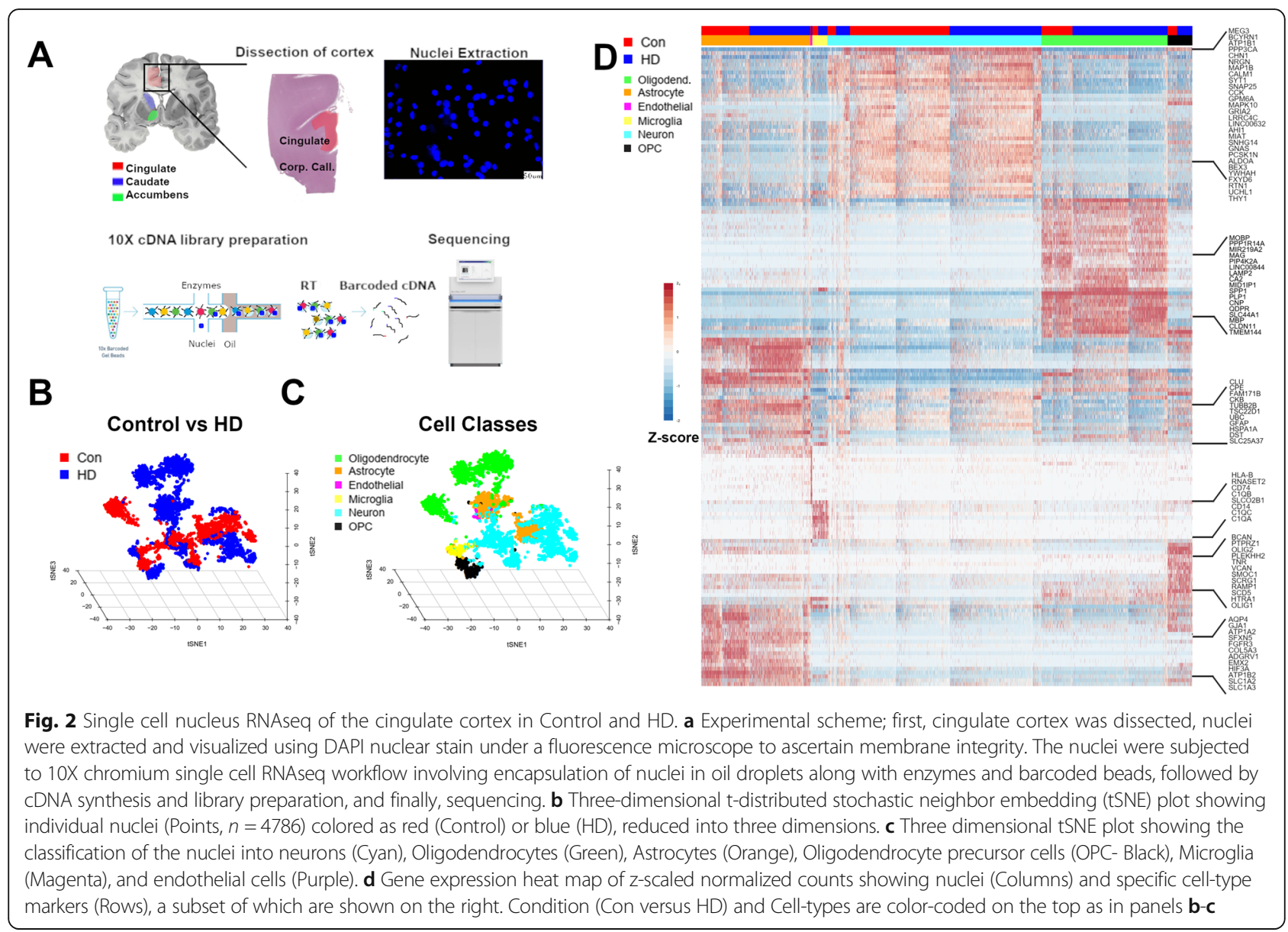

\section{Astrocytes in the HD cingulate cortex}

To investigate astrocytic changes in HD in the cingulate cortex, we focused on astrocytic nuclei (1064) and projected these nuclei into the tSNE space (Fig. 3a). Control and HD astrocytic nuclei were separated in the tSNE space. We next performed sub-clustering using consensus k-means clustering in the SC3 package in R and identified six clusters, three control and three HD astrocytic, with minimal overlap between the conditions (Fig. 3b and Additional file 8: Video S1). We next identified cluster-specific markers using the SC3 package pairwise gene Wilcoxon signed test with p.value of 0.05 , Holm's method for p.value adjustment, and area under receiver operator curve (AUROC) 0.65 (Fig. 3c). We then examined differentially expressed genes in HD astrocytes versus control astrocytes. One thousand six hundred fortyfive genes were downregulated in HD while 607 genes were increased (exact $p$ value and Adjusted BenjaminiHochberg adjusted $p$.value $<0.05$ ). Analysis of enriched gene ontology terms and Reactome pathways showed the top gene ontology molecular function terms included terms related to heat shock protein binding, unfolded protein binding, MHC binding, and metal ion binding, while enriched Reactome pathways included response to metal ions, metallothionein binds metals, and Heat-shock factor 1 (HSF-1) dependent transactivation (Fig. 3d). In contrast, molecular function gene ontology terms enriched in downregulated astrocytic genes included terms related to symporter activity, amino acid binding, and neurotransmitter:Sodium symporter, while enriched Reactome pathways included Notch signaling and cholesterol biosynthesis (Fig. 3e). A list of differentially expressed genes in HD versus control astrocytes as well as the full results of GO term enrichment analysis are provided in Additional file 9: Table S2A-B. A selected list of astrocytic sub-cluster marker genes is provided in Table 2. The full results of differential gene expression between each sub-cluster against all other sub-clusters is provided in the Additional file 4: Supplementary data.

Since Liddelow et al. [27] showed that astrocytes in the HD caudate nucleus expressed markers of a putative neurotoxic state (A1 state), we compared the cingulate cortex to the caudate using an antibody to $\mathrm{C} 3$ - a marker of the A1 astrocytic state. We confirmed that numerous cells morphologically consistent with astrocytes in the 

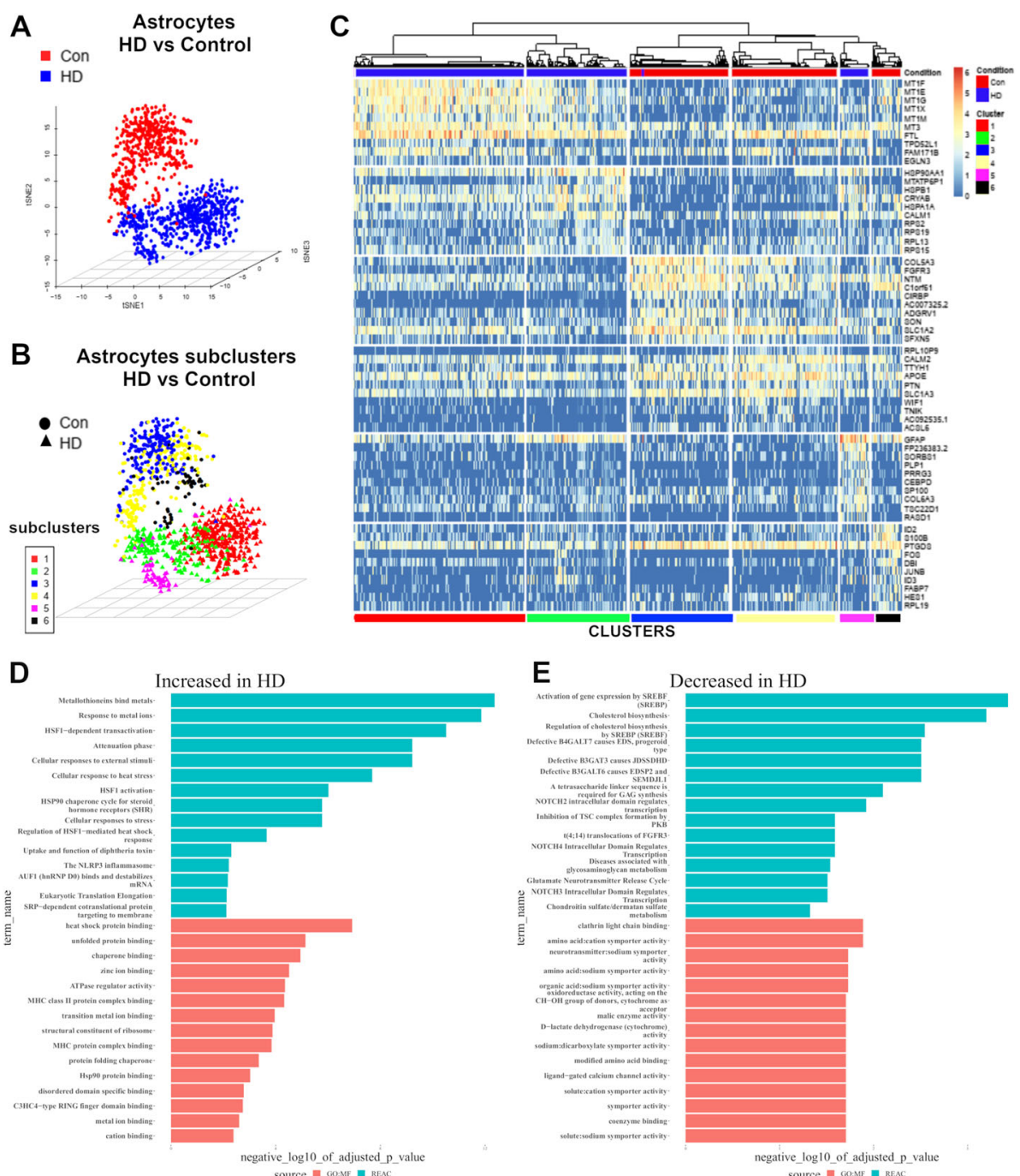

Fig. 3 Transcriptomic analysis of astrocytic nuclei in control and HD. a Three-dimensional t-distributed stochastic neighbor embedding (tSNE) plot showing astrocytic nuclei ( $n=1064-469$ control and 595 HD) colored as red (Control) or blue (HD), reduced into three dimensions. $\mathbf{b}$ Three dimensional tSNE plot showing the classification of the astrocytic nuclei into 6 sub-clusters using consensus k-means clustering (sc3 package). c Gene expression heat map of cluster markers showing nuclei (Columns) and specific cell-type markers (Rows). Condition (Con versus HD) and Cell-types are color-coded on the top and bottom, respectively. Cluster-specific gene markers were identified using Wilcoxon signed rank test comparing gene ranks in the cluster with the highest mean expression against all others. p-values were adjusted using the "Holm" method. The genes with the area under the ROC curve (AUROC) $>0.65$ and p-value $<0.01$ are shown as marker genes. $\mathbf{d}$-e $\mathrm{GO}$ term analysis of differentially expressed genes in $\mathrm{HD}$ versus control astrocytes identified using EdgeR likelihood ratio test with an adjusted p.value of 0.05 . Significantly enriched $\mathrm{GO}$ terms at Benjamini-Hochberg false discovery rate of 0.05 were identified. Selected Reactome pathways and Molecular function $\mathrm{GO}$ terms which are increased in HD astrocytes (d) and decreased in HD astrocytes (e) are shown

caudate nucleus of HD grades (III and IV) were C3 positive compared to controls. Double immunostaining for C3 and GFAP (Additional file 10: Figure S3D) as well as C3 and LN3 (microglial marker), showed immunopositivity for C3 in GFAP-positive astrocytes, and minimal immunopositivity in microglia (Additional file 10: Figure S3C, D). In contrast, immunostaining in the cingulate cortex showed C3 labeling of neurons in controls and HD cases, but no astrocyte labeling, with no clear differences (Additional file 10: Figure S3A-B). Together, these findings confirm previous reports of the A1-astrocytic marker C3 immunopositivity in striatal astrocytes in HD, but, in contrast, show minimal astrocytic labeling with $\mathrm{C} 3$ in the HD cingulate cortex.

\section{Multiple genes are differentially correlated in HD and control cortical astrocytes}

We examined the gene-expression heatmap for the 6 astrocyte clusters (Fig. 3c). Clusters 1, 2, and 5 were from HD cortex, while 3, 4, and 6 were from control 
cortex. It is apparent that the control clusters 3 and 4 express high levels of genes associated with protoplasmic astrocytes, like FGFR3, GLUL, and SLC1A2, although there are differences from cluster to cluster and from cell to cell, and control cluster 6 shows high levels of GFAP. The HD clusters 1 and 2 express high levels of MT genes (MT1F, MT1E, and MT1G.), as well as GFAP, although MT genes were generally more highly expressed in cluster 1 than in cluster 2 or cluster 5 and GFAP was more highly expressed in cluster 2 and 6 than in cluster 1 . We provide the full differential gene expression between astrocytic clusters in Additional file 4.

Given this heterogeneity of gene expression, we set out to identify how astrocytic genes are co-regulated. We performed differential gene correlation analysis between the top $5 \%$ most highly expressed astrocytic genes on average using the DGCA R package. The results revealed the MT genes were highly correlated with each other, and the correlation was significantly stronger in HD astrocytes than in control astrocytes (Fig. 5a). Moreover, there were differences in the correlation of many gene pairs between control and HD astrocytes. For instance, multiple MT genes were negatively correlated with GFAP, including MT1G, MT1F, MT1E, and $M T 2 A$ in HD astrocytes. However, these gene pairs were either not correlated or only minimally positively correlated with GFAP in control nuclei (Fig. 5a and Additional file 11: Table S3). As an example, a scatter plot showing the expression of the GFAP and MTIG in control and HD astrocytes reveals that the two genes are negatively correlated (Fig. 5b); with Pearson correlation coefficient of 0.21 in $\mathrm{HD}$ and 0.18 in control astrocytes (adjusted $p$ value of difference $<0.0001$ ). In contrast, MT genes $M T 1 F$ and MT2A were positively correlated with genes associated with protoplasmic astrocytes including SLC1A3 in HD, but negatively correlated in control astrocytes (Pearson correlation coefficients of 0.14 (HD) and - 0.14 (Control) for $M T 2 A$ - adjusted p value of difference < 0.01 , and 0.1 (HD) and - 0.14 (Control) for MT1F -adjusted $\mathrm{p}$ value of difference $<0.05$ - Additional file 11: Table S3).

These analyses of HD and control astrocytes reveal a heterogeneity of gene expression phenotypes in both the $\mathrm{HD}$ and control populations that would not have been detected without snRNA-seq. The heterogeneity also adds a layer of complexity both to the populations of astrocytes in control cortex as well as to the populations of astrocytes reacting to a disease.

\section{Gene co-expression network analysis in HD and control astrocytes}

Next, we aimed to identify gene networks in which subsets of astrocyte genes were linked to each other. We used the Multiscale Embedded Gene Co-Expression Network Analysis (MEGENA [49]). A usual application involves building gene networks in the control condition, and examining how the network structure changes in the test condition. However, given that control and HD astrocytes clustered separately, and there were significant gene expression changes between the two conditions, we decided to use all astrocytes from both conditions for gene network analysis. This allowed discovering both condition-specific and shared gene networks. Application of the algorithm produced 15 gene clusters (modules). The network hierarchy structure is shown in Fig. 5c. The modules on the same arm of the hierarchy tree shared many genes. For example, modules 3 and 10, modules 22 and 26, as well as modules 9 and 20, shared many genes (Additional file 12: Table S4A-B, which details all the module genes and hub genes). Examples of modules are shown in Fig. 5d.

We then correlated each module with one or more of the 6 astrocyte clusters. To indicate the functional characteristics of the modules, we used Gene Ontology term enrichment analysis to ascribe "Molecular Function" gene ontologies that were enriched in each of the modules. Modules 9 and 20 were mostly enriched in the HD cluster 1 (compare Figs. 5e and f) and have genes associated with molecular function gene ontology terms like amyloid beta binding and low-density lipoprotein particle receptor binding - genes known to be associated with reactive astrocytes in Alzheimer's disease [31, 47]. Module 7 was also most enriched in cluster 1 and has genes enriched for GO terms related to metal binding (see above regarding MT genes). Modules 6 and 16 are enriched in the HD cluster 5 , and include genes enriched for GO terms related to epithelial-mesenchymal transition, RNA-mediated gene silencing, and DNA binding (Fig. 5e-f and Additional file 12: Table S4C-D). Modules 4, 12,22 , and 26 are enriched in control cluster 3 and have genes enriched in $\mathrm{GO}$ terms related to regulation of neurotransmitter levels, autophagy, sodium ion transport (Module 12), and cell-cell signaling. Module 5 was most enriched in control cluster 6 and has genes involved in protein transport, localization, and biosynthesis. Module 17 was enriched in control cluster 4 and has genes enriched for GO terms related to nucleotide triphosphatase, pyrophosphatase, and hydrolase activity. Shared between HD cluster 2 and control cluster 4 are Modules 3 and 10, which have genes enriched for GO terms related to aerobic respiration, nucleotide metabolism, and ATP biosynthesis. Of interest, Module 13 was enriched in control astrocytic clusters Astrocyte_3 and Astrocyte_4 (Fig. 5f), and showed enrichment for GO terms relating to fatty acid biosynthetic process, fatty acid binding, longchain fatty acid biosynthetic process, and prostaglandin biosynthetic process (Additional file 12: Table S4C-D). Conversely, Module 20 was enriched in HD cluster Astrocyte_1 and less so in control cluster Astrocyte_4. This module was enriched for GO terms relating to lipid metabolic process, phospholipid binding, and lipid transport 
(Additional file 12: Table S4C-D). The top gene ontologies per module are provided in Additional file 12: Table S4D. Thus, in most cases, the network modules appear distinct for either control or HD astrocytes, although there is some overlap. Molecular function analysis further highlights the heterogeneity of both control and HD astrocyte populations.

\section{Validation of astrocytic gene expression changes in HD}

The snRNASeq analysis revealed significant differences in astrocyte gene expression between HD and controls. We wanted to validate these gene expression differences and therefore conducted immunohistochemical and in situ hybridization studies in HD and control cingulate cortices (Fig. 4). To quantify astrocytic activation in the HD cingulate cortex we measured the cell density of GFAP immunopositive cells (Fig. 4a). We found that the number of GFAP immunopositive cells was increased in grade III/VI HD compared to control (Fig. 4c and see Additional file 4: Methods). We also confirmed that the GFAP transcript levels were indeed increased in the HD cingulate cortex using rt-qPCR (Fig. 4d). Moreover, we quantified the optical density of the GFAP signal in fluorescently immune-stained sections as a surrogate for protein content in each astrocyte (Additional file 4: Methods). We found that GFAP levels as measured by GFAP signal optical density was increased in HD astrocytes (34.98 +/- 1.92 arbitrary units; mean +/- standard error of the mean (sem)) compared to controls (19.83 $+/-1.95$ arbitrary units; mean $+/-$ sem, $p<0.001$ ).

Because HD is caused by HTT mutations, we examined HTT transcript levels in astrocytes in control and HD patients. We found that levels of HTT RNA were reduced in HD astrocytes (Additional file 4: Results). We next wanted to ascertain whether HTT accumulates in HD astrocytes. Therefore, we double stained sections with the GFAP antibody and an antibody for wild-type HTT to detect HTT aggregates in astrocytes. We found that HTT does indeed aggregate in HD astrocytes (Fig. $4 \mathrm{~b})$ mainly in layers V and VI. These data suggest that astrocytic reactivity in HD may at least be partly cellautonomous, or possibly that astrocytes phagocytose HTT aggregates from the surrounding neuropil.

We explored further the astrocyte heterogeneity indicated by the transcriptional data by using multiple label immunofluorescence on a larger group of control $(n=3$, two of which were cases on which we did not do snRNASeq) and HD ( $\mathrm{n}=3$, two of which were cases on which we did not do snRNASeq) cingulate gyri. Here we used antibodies to ALDH1L1, as a general astrocyte marker, to MTs, as a marker for early reactive astrocyte clusters, and to GFAP (Fig. 4e and Additional file 13: Figure S8). The results showed more HD astrocytes coexpressed MT; with $45.57+/-25.58 \%$ of GFAP+ or
ALDH1L1+ astrocytes co-labeling with MT compared to $10.24+/-7.64 \%$ of control astrocytes (mean $+/-$ standard deviation, $p=0.043$, one-sided $\mathrm{t}$-test). Additionally, we found that levels of MT were also increased in HD astrocytes compared to control astrocytes. More specifically, we measured MT fluorescent signal (optical density) and found it was increased in HD astrocytes (24.33 $+/-1.76$ arbitrary units; mean $+/-$ sem) compared to controls $(12.3+/-1.14$ arbitrary units; mean $+/-$ sem, $\mathrm{p}<0.001$ ). These results show that more HD astrocytes expressed MT and that they expressed higher levels of MT, confirming the snRNASeq results. Note that these $\mathrm{MT}+/ \mathrm{GFAP}+$ astrocytes are likely to represent HD cluster 2, which, as noted above, expresses the highest levels of MT genes.

To identify cluster 1 astrocytes, which express little GFAP but relatively elevated levels of MT, we used ALDH1L1 in triple immunofluorescent stains with GFAP and MTs. We found that astrocytes in HD cluster 1 are indeed identifiable in the HD cingulate cortex, with low to undetectable GFAP levels but immunopositivity for ALDH1L1 and MT (Additional file 13: Figure S8C). These astrocytes are in contrast to cluster 2 astrocytes which show triple-immunopositivity for ALDH1L1, GFAP, and MT (Additional file 13: Figure S8B, C and Fig. 4e). Triple labeling also detected astrocytes that stained for ALDH1L1 but not for either MTs or GFAP (Additional file 13: Figure S8A). These represent astrocyte clusters 3 and 4 , which are far more frequently found in control than in HD cortex. Finally, we found rare examples of astrocytes in control cortex that were examples of cluster 6, showing ALDH1L1+/MT- or low/ GFAP+ (Additional file 13: Figure S8A).

Interestingly, we noted co-expression of proteolipid protein (PLP-1), a myelin gene, and GFAP in HD cluster 5 (Fig. 3c). We verified this in tissue sections using in situ hybridization. The results showed that while PLP-1 and $M B P$ were co-expressed in oligodendrocytes, GFAP and $P L P-1$ were co-expressed in a subset of astrocytes in the HD cortex ( $n=5$ cases, Fig. $4 \mathrm{f})$. In contrast, only one case from 4 controls showed rare PLP-1 co-expression with GFAP in the cortex. Notably, gene expression analyses did not reveal other oligodendrocyte genes, including OPALIN, MOG, MAG, CA2, CD82, OMG MYRF, SOX10, and NAFSC, implying that the GFAP+ astrocytes were not upregulating a coordinated transcriptional program of oligodendrocyte maturation.

\section{Three astrocytic states of reactivity in HD}

A simplified view of astrocytic gene expression profiles reveals patterns based on the expression of protoplasmic astrocytic genes, reactive astrocytic genes, and MTs. We performed a supervised classification of astrocytes based on expression of MT2A, GFAP, and SLC1A2.The results 


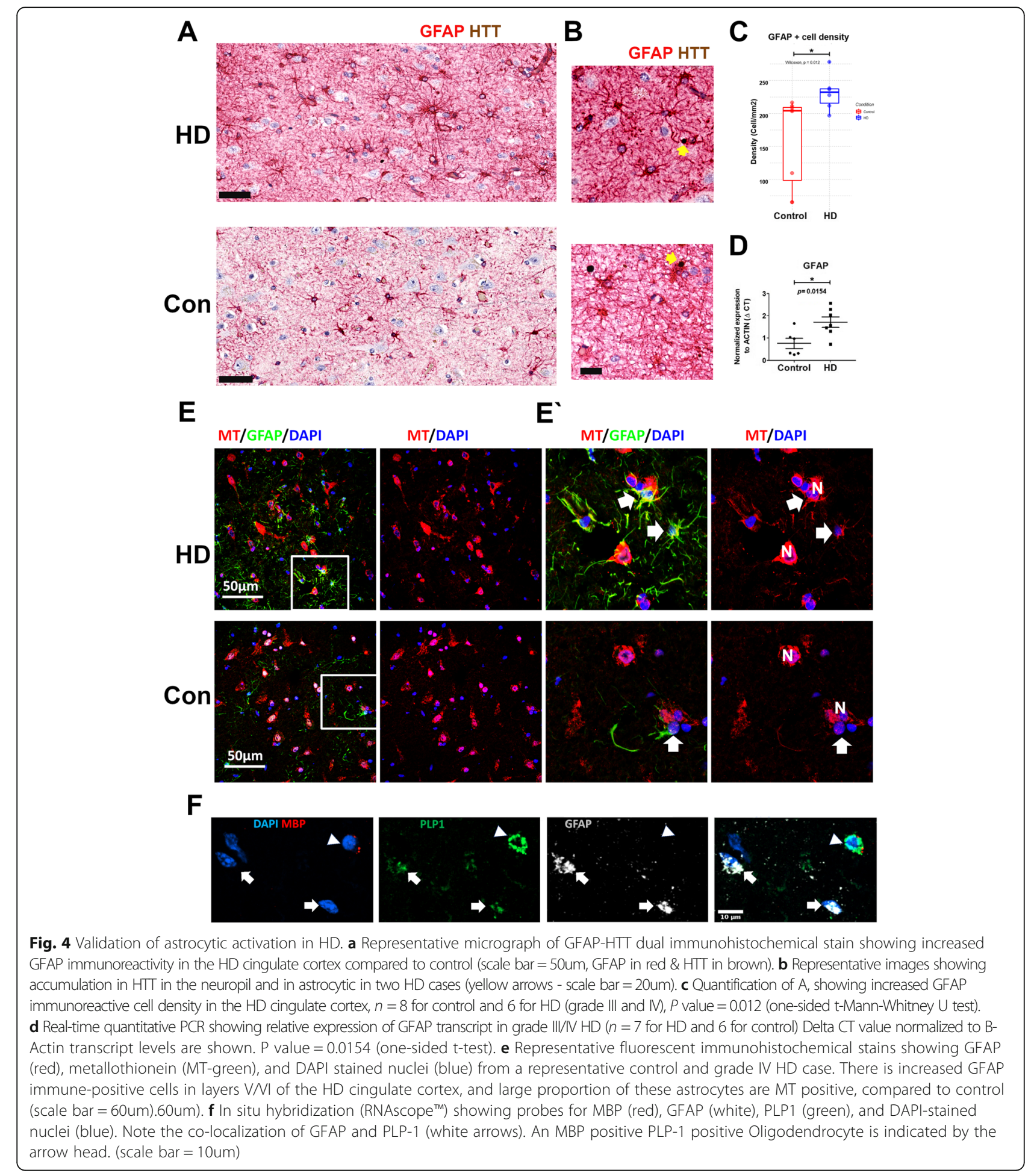

show three distinct astrocytic states in HD and control astrocytes. Astrocytes with low levels of $M T 2 A$, low GFAP and high SLC1A2 were classified as Quiescent. Astrocytes with high levels of $M T 2 A$, low GFAP and high SLC1A2 were classified as state-1Q - for quiescent astrocytes with early reactive features. Astrocytes with high levels of $M T 2 A$, high GFAP and lowSLC1A2 were classified as state2-R - for reactive astrocytes with high $M T$ s. Astrocytes with lower levels of $M T 2 A$, high GFAP and lowSLC1A2 were classified as state3-R - for reactive astrocytes with lower MTs (Fig. 6a). As expected, unclassified states are also seen. These represent astrocytes 
that did not meet any of the classification criteria ("unknown" type of astrocytes), or astrocytes that met more than one category ("ambiguous"). Possibly, these may represent transitional states. We then examined the relative preponderance of these astrocytic states in control versus HD (Fig. 6b). We found that the Quiescent state is abundant in control astrocytes $(49.3 \%)$, but low in HD (1.6\%). In contrast, states1-Q and 2- $\mathrm{R}$ were more abundant in HD (37.4 and 14.0\% in HD versus 21 and 1.4\% in control). State3-R was relatively low in both conditions, but more abundant in HD than control $(7.9 \%$ versus $2.5 \%$ ). As noted above, we found examples of all 3 states by immunofluorescence in all HD cases we examined. The presence of these states is therefore a more general phenomenon than what we have found only by transcriptomics.

The states of astrocyte reactivity correspond to specific astrocytic clusters (Fig. 6c). For example, in contrast to the Quiescent astrocytic state, which was comprised of almost entirely control clusters, astrocytic state-1Q was mixed, with a major contribution from HD cluster 1 and smaller contributions from other astrocytic clusters. Moreover, state-2-R was primarily composed of HD clusters 1 and 2, while state3-R was largely comprised of HD clusters 2 and 5 . In summary, we find three reactive astrocytic states based on the expression of GFAP, SLC1A2, and MTs (Fig. 6c). These states relate to reduction of expression of protoplasmic genes (e.g. SLC1A2, FGFR3, GLUL), and increased MTs in one reactive subset of astrocytes versus a preponderance of GFAP expression in another reactive subset.

It is difficult to relate these states to the A1 versus A2 astrocytes. For starters, none of the "states" or clusters in the cingulate cortex showed $C 3$ expression. Moreover, only 25 of 39 astrocytic genes described by the Barres group were detected by scnRNAseq, and only 3 were significantly increased in HD (GFAP, SRGN, and CD14). Taken together, we cannot ascribe an A1 versus A2 phenotype to cingulate HD astrocytes.

\section{Gene expression analysis of non-astrocyte cells}

Although we have focused on astrocytes in this report, snRNAseq provided gene expression patterns of all other cell types, neurons, microglia, oligodendrocytes, oligodendrocyte precursors, and endothelial cells. We found differences in gene expression patterns for both neurons and microglia that distinguished HD from control cortex, and present these findings in the Additional file 4: Supplement. For oligodendrocytes we have evidence that the heterogeneity of cells of the oligodendrocyte lineage is increased in HD, with a shift to less mature phenotype (manuscript in preparation).

\section{Discussion}

The HD cingulate cortex contains multiple reactive states of astrocytes

One of the most notable findings in this study is that there are multiple states of astrocyte reactivity, rather than a single reactive state. This is not surprising, given that $\mathrm{HD}$ is a long-term, progressive disease in which the neuropathology does not evolve in synchrony. HD cluster 1 showed high levels of $M T$ gene expression, and relatively low GFAP expression. In contrast, HD cluster 5 showed high GFAP expression and relatively low $M T$ gene expression. Intermediate levels of MTs and GFAP were the hallmark of HD cluster 2. In all HD astrocytes, the mRNA levels of glutamate transporters SLC1A2 and SLC1A3 expressed in quiescent astrocytes were reduced, implying that HD astrocytes downregulate genes central to some critical astrocytic functions. It is tempting to speculate that these states reflect a temporal sequence of astrocyte reactivity, and that one progresses to another over time, so that the highest GFAP astrocytes that have lost many normal genes as well as $M T$ s represent an end stage of reactivity. We do not have definitive evidence that this is so. However, when we analyzed striata from these same brains we saw a greater proportion of astrocytes in this possible end stage state (unpublished data), given the far high degree of neuronal degeneration in striatum than cingulate. Additional evidence from examining HD cases with different grades of severity as well as evidence from experimental models such as astrocyte lineage tracing experiments in conditional HD mice models are needed to query this possibility further. Alternatively, difference in astrocytic reactive states may reflect spatial differences in astrocyte localization in cortical layers with variable susceptibility to neurodegeneration, such as susceptible layers III, V, and VI versus resilient layer II [7, 45].

The presence of high expression of $M T$ genes, as well as anti-oxidant genes in HD cluster 1 may suggest that this astrocyte "state - State 1Q" is protective. In fact, these astrocytes may be protective of neurons and also of themselves, an "astro-protective" state. Resolving how this state relates to the A2 state is an interesting matter, however, it is not possible to evaluate this putative relationship without additional experimentation. Are both states underpinned by the same molecular signaling pathways, such as STAT3 activation for example? Or are these states divergent? Such questions are intriguing and will be the subject of future work.

\section{Classifying astrocytic reactivity in the human brain}

Our data showed that both A1 and A2 genes were increased in bulk RNAseq samples, which is consistent with recent whole tissue RNASeq data from Diaz-Castro et al. [7]. However, these A1 versus A2 states did not 
become more clear on the single cell level. We anticipated finding expression of A1 genes in a subset of astrocytes, and A2 in another. However, we found that only three genes were differentially expressed in HD astrocytes (GFAP, CD14, and SRGN). Thus, applying the A1 versus A2 classification is not appropriate in the HD cingulate cortex. What is apparent from our data is that protoplasmic genes, including genes related to calcium signaling (Fig. 3e), are downregulated in reactive astrocytic states in HD. This data is consistent with DiazCastro et al. [7], where the authors found that there are common genes that are downregulated in both striatal murine astrocytes and post-mortem human striatalderived microarray data, and that these genes were mostly related to calcium ion transport, G-protein coupled receptor signaling, and glutamate receptor signaling. Thus, we contend that viewing astrocytosis in HD in terms of loss of astrocyte function, and gain of potentially neuroprotective phenotypes, is a useful way to view reactive astrocyte states in HD. Note, however, that the whole tissue RNASeq analysis [7] does not reveal astrocyte transcriptional heterogeneity and would not have defined different astrocyte "states."

\section{The control cingulate cortex also contains a heterogeneity of astrocytes}

Not only did we find a heterogeneity of astrocyte phenotypes in the HD cortex, we also found variation in astrocyte populations in the control cortex, based on the gene expression profiles. For example, astrocyte cluster 6 appears different from both 3 and 4 in that it has higher levels of GFAP, CRYAB, FOS, JUNB, ID2 and ID3 and lower levels of GLUL, SLC1A2, and SLC1A3, indicating that astrocytes of this group have downregulated protoplasmic astrocyte genes and upregulated "reactive" genes. Clusters 3 and 4 also show differential gene expressions. The heterogeneity of astrocyte gene expression must be validated and explored in depth in the future. However, it does raise the possibility that there is a significant amount of astrocyte heterogeneity in the control cortex. We do not know if this heterogeneity is fixed, or whether gene expression variations are transient, depending on such factors as neuronal activity or local blood flow, for example. Furthermore, the presence of astrocytes with "reactive" markers in a "control" cortex may well reflect an individual's age, past medical history or terminal illness, even in patients without histories of neurological diseases.

\section{HD astrocytes upregulate metallothionein genes}

$M T$ genes are thought to confer neuroprotection. Levels of MTs are increased in multiple injuries including ischemia, heavy metal exposure, infection, and neurodegeneration in $\mathrm{AD}$ [46]. Mathys and colleagues investigated the frontal cortex of human late onset Alzheimer disease using snRNAseq and showed expression of $M T 2 A$, $M T 1 G$, and MT1E in astrocytic cluster Ast1 [31]. Mice deficient in $M T 1 / 2$ show impaired repair and wound healing after freeze injury, with increased microglia/macrophage infiltration, astrocytosis, and apoptosis [40]. Neuronal survival was compromised in MT1/2 knockout mice after middle cerebral artery occlusion [59]. Conversely, mice overexpressing MT1 showed increased resilience to ischemic damage in the same model of injury [56]. The evidence suggests that upregulation of MTs maybe a protective response to injury and we propose that it may be so in HD astrocytes. As we have noted above, upregulation of MTs may not only be neuroprotective but also astro-protective. A recent study of HD is also consistent with upregulation of $M T$ genes. Lin et al. [29] examined transcriptional signatures in the motor cortex (BA4) of grade 2-3 HD showed that GO terms involved in Response to Cadmium Ion (GO0071276), Zinc ion (GO0071294, GO0010034) were increased, while Cholesterol synthesis was reduced (e.g. GO0006695). These data are consistent with our results in HD astrocytes and suggest that upregulation of $M T \mathrm{~s}$ in reactive astrocytes is a pan-disease reactive response. It is known that oxidative stress is a characteristic of the HD brain [50]. The fact that MTs are involved in combating oxidative stress, and that they are upregulated in HD astrocytes, impart a potential neuroprotective role for this phenomenon, perhaps an A2-like state.

On another note, our data highlights fatty acid synthesis and lipid metabolism as key processes that are differentially regulated in HD astrocytes. We find that HD astrocytic clusters had downregulated genes that are associated with fatty acid and prostaglandin biosynthesis. Indeed, the gene PTGSD (Prostaglandin D2 Synthase) and FASN (Fatty Acid Synthase) were reduced in HD astrocytic clusters (Fig. 3c and Additional file 9: Table $\mathrm{S} 2 \mathrm{C}$ ). Moreover, cholesterol biosynthesis was reduced in HD astrocytes (Fig. 3e). In contrast, lipid binding and metabolism were processes enriched in genes characteristic of HD astrocytic cluster 1 (Module 20 - Fig. $5 \mathrm{f}$ and Additional file 12: Table S4C-D).

\section{Astrocyte modules}

Analyzing astrocytic gene expression in terms of gene modules provides a useful way to examine correlated genes and hub genes. The latter may be prioritized in functional studies such as overexpression and knockdown/knockout studies. We constructed the modules using all astrocytes, from control and HD brains. Had we constructed modules from HD and control astrocytes separately, the network structures would have been different. For example, module_26, a module harboring many protoplasmic genes, would likely have been devoid of $C R Y A B$, a gene upregulated in reactive states. 
A

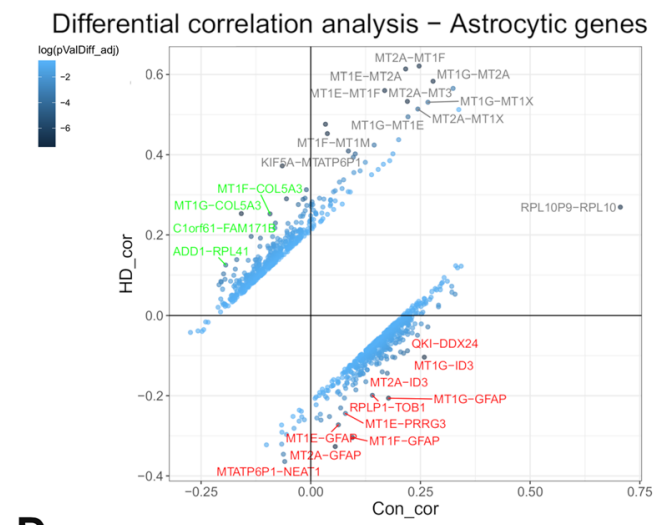

B

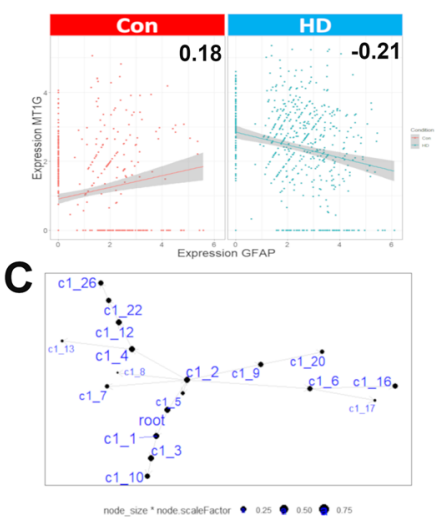

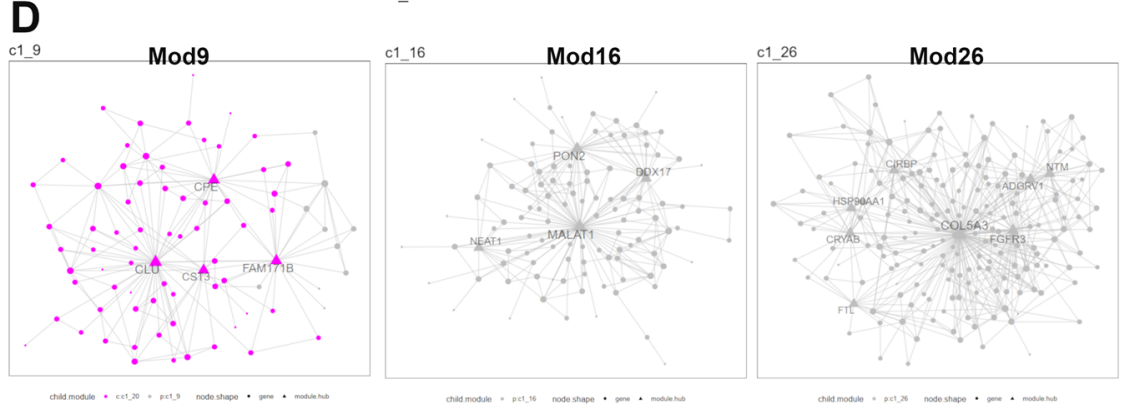

E

Molecular Function GO term analysis of astrocytic modules

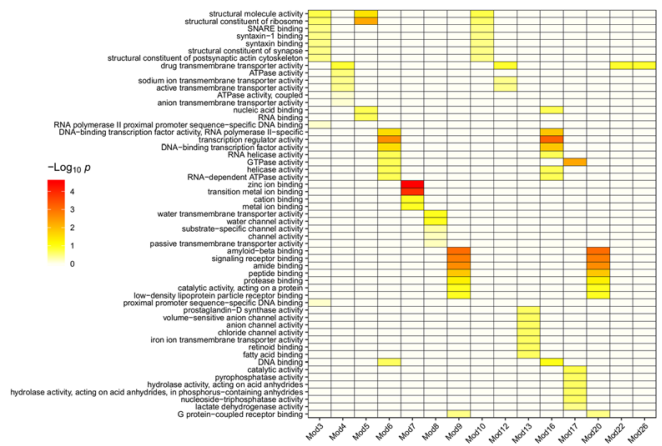

F Astrocytes_HD_vs_con_GSVA

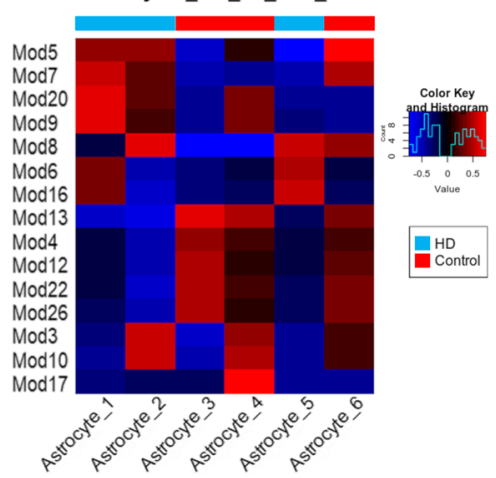

Fig. 5 A deeper look into astrocytic gene regulation in HD. a Differential gene correlation analysis of the top 5\% of genes in astrocytic nuclei by mean normalized expression (856 genes). Pearson correlation coefficients are shown and empirical p-values were calculated by a permutation test (100 permutations) in DGCA package in R. Top gene-pairs that are significantly differentially correlated between HD and control astrocytes are labeled. $\mathbf{b}$ Scatterplot of normalized expression of GFAP and MT1G (A representative metallothionein gene) in Control (left panel) and HD (Right panel) astrocytic nuclei. Pearson correlation coefficients are shown and are significantly different between control and HD. c Hierarchy structure representing the astrocytic gene modules/networks (labeled here as $\mathrm{Cl}{ }_{-} 3, \mathrm{C1} \_5, \ldots$ etc) as the output using Multiscale Embedded Gene Co-Expression Network Analysis (MEGENA). Clusters on the same line are more related to each other than clusters on different lines. $\mathbf{d}$ Representative gene modules/networks, illustrating modules 9, 16, and 26. Hub genes are shown as triangles, genes as nodes, different colors represent different networks in the same graph (Left panel - Mod9). e Gene Ontology term enrichment analysis showing representative "Molecular Function" gene ontologies enriched in astrocytic gene modules. $\mathbf{f}$ Gene set variation analysis showing enrichment scores of modules in different astrocytic clusters, condition is shown as colored bars on the top

The strength of our approach; combining both control and HD astrocytes, is that it allows us to discover both HD and control modules as well as shared modules, such as module_3 (shared between clusters Astrocyte_2 and Astrocyte_4). Genes in this module relate to oxidative phosphorylation and Wnt signaling (Fig. 6c).

A summary of gene expression differences between astrocytic clusters in different states of reactivity summarized as modules is shown in Fig. 6c. Control astrocytic clusters 


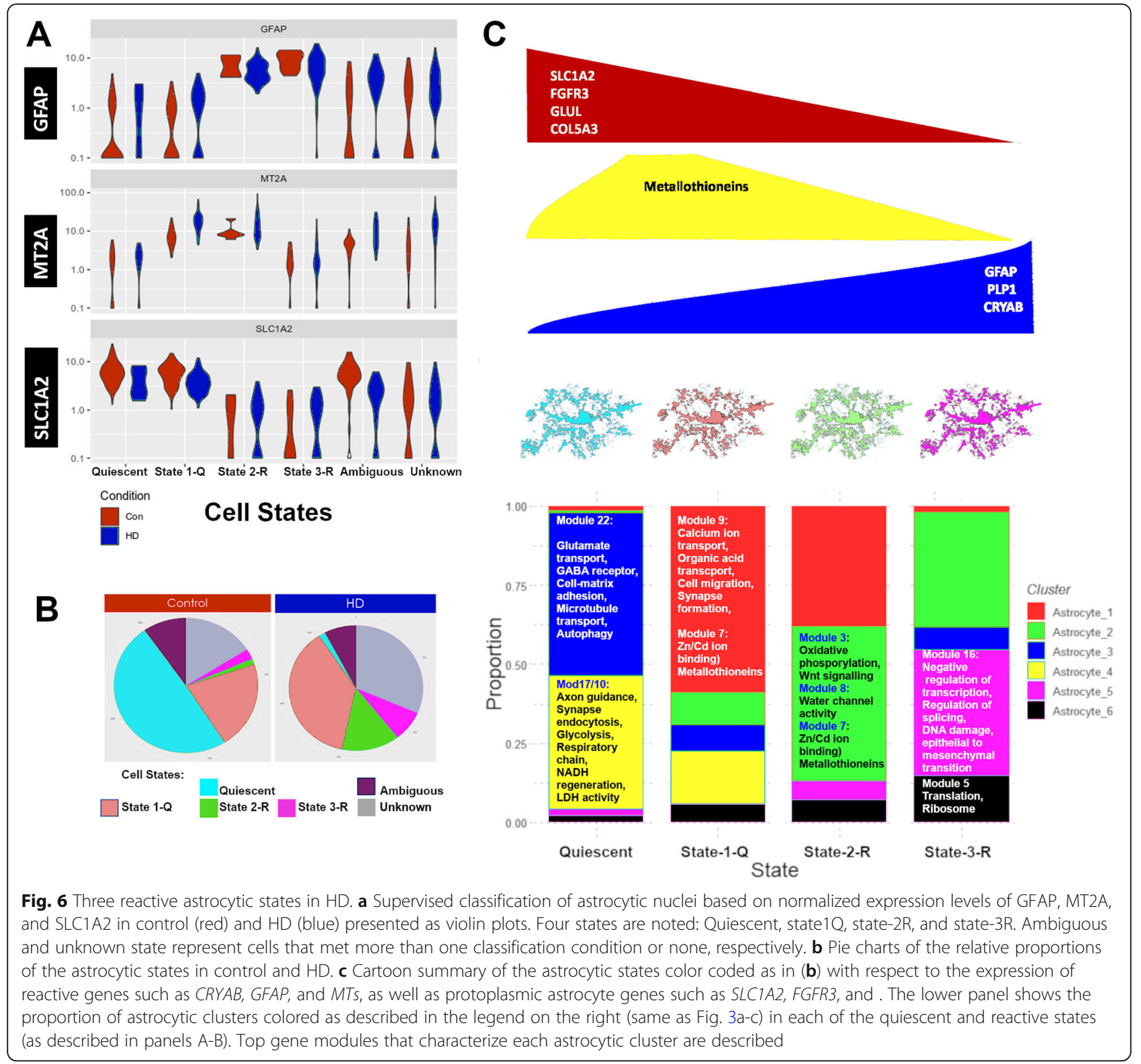

Astrocyte_3 and Astrocyte_4 are represented in quiescent and state1-Q, and are enriched in genes described by modules 22, 28, 10, and 17, which harbor genes involved in glutamate transport, GABA receptors, autophagy, glycolysis, cell respiration, and synaptic function. HD cluster 1 is represented in states 1-Q and 2-R, while HD cluster 2 is represented in states $2-\mathrm{R}$ and 3-R. Both clusters are enriched in genes of module_7 (MTs). Conversely, HD cluster 5 is represented mainly in state $3-\mathrm{R}$ and is enriched in module 16 , harboring genes involved in mesenchymal differentiation, DNA damage, and negative regulation of transcription. Overall, we present complementary views to understand gene expression changes in HD astrocytes in the cingulate cortex, using unsupervised clustering, supervised classification, and gene network analysis.
Is HD astrocyte pathology cell-autonomous?

Whether the astrocytic reactivity in HD is a cell autonomous reaction or a secondary reaction to neurodegeneration, or both, is yet to be determined. We provide evidence that HTT indeed accumulates in astrocytes in the HD cortex. These HTT aggregates are seen in a minority of HD astrocytes, primarily in layers V and VI of the cingulate cortex, where neurodegeneration is most pronounced [13]. We also provide evidence that neuronal loss and dysfunction is evident in the HD cingulate cortex (See Additional file 4: Supplement).

Thus, astrocytic reactivity in HD may indeed be secondary to neuronal loss. However, the astrocytic dysfunction may also contribute to neuronal loss and dysfunction. HD transgenic mouse studies that target 
$m H T T$ to astrocytes exhibit neuronal loss, suggesting that mutant HTT expressed in astrocytes alters homeostatic functions and precipitates neuronal dysfunction and loss. Restoration of astrocytic function ameliorates the HD phenotype and prolongs survival in vivo [53]. Additional evidence from the HD mouse model R6/2 indicates that chimeric mice engrafted with control human glia including astrocytes exhibited slower disease progression. Conversely, control mice engrafted with HD glia including astrocytes exhibited impaired motor coordination [3]. Thus, it is possible that astrocytic dysfunction in HD may be partly cell autonomous and partly secondary to neuronal dysfunction. In support of this notion, Diaz-Castro et al. [7] showed that silencing of $m H T T$ expression in murine striatal astrocytes reduced the accumulation of mHTT in medium spiny neurons. Future studies to correlate the cortical locale of reactive HD astrocytes with the transcriptional phenotype may also help to answer this question.

Our data on neuronal gene expression profiles on the bulk RNAseq level and the single cell level as well as neuronal estimates show major transcriptional and cellular alterations. First, we show that large (pyramidal) neurons are depleted in the HD cingulate (as verified by histopathologic quantification). Second, we show that neuronal genes are downregulated in bulk RNAseq HD samples. For example, pathways involved in glutamate, GABA, neuropeptide Y neurotransmission were downregulated in HD. Finally, our snRNAseq show that glutamatergic neurons, in contrast to interneurons, cluster separately between control and HD. Moreover, neurons upregulate metallothioneins and pathways involved in heat-shock response, voltage-gated ion channels, and protein misfolding. Therefore, neuronal loss and dysfunction is evident in the HD cingulate cortex and may underlie astrocytic reactivity, but it could also be at least partially secondary to astrocytic dysfunction.

\section{Upregulation of inflammatory genes in the HD cingulate cortex}

The bulk RNAseq analysis showed upregulation of a number of inflammatory genes (see Additional file 4: Supplementary Data and Additional file 14: Figure S6). Our findings highlight the activation of the innate immune system including toll-like receptor pathways. Multiple interleukin signaling pathways were enriched in HD including IL-4, IL-13, and IL-10. Of note, we found IL-10 to be significantly upregulated in HD versus controls. IL-10 has been previously reported to be elevated in the serum and CSF of HD patients [4].

\section{Conclusions}

In summary, we show using snRNAseq from postmortem human HD cingulate cortex, that astrocytic reactivity can be described in three states with different levels of GFAP, metallothionein genes, and quiescent protoplasmic genes. Immune pathways were prominently enriched in the HD cortex, although we were only able to detect them using bulk RNAseq. There were significant gene expression differences between HD and control neurons, particularly in the excitatory neuron populations, along with neuronal loss. We have made our snRNAseq data available using an interactive web application found here (https://vmenon.shinyapps.io/hd sn_rnaseq/).

\section{Supplementary information}

Supplementary information accompanies this paper at https://doi.org/10. 1186/s40478-020-0880-6.

\begin{abstract}
Additional file 1. Outline of the snRNAseq analysis pipeline. Briefly, filtered raw un-clustered data is pre-clustered using a shared nearest neighbor algorithm. The pre-clusters are classified into cell classes/ lineages using gene set enrichment analysis for specific lineage genes and examining GO terms of the top pre-clusters markers. Next, mixed pre-clusters are identified and the cells in these pre-clustered are reclassified based on the cell-specific lineage-scores (Cell classifier tool). Next, clusters of the same lineage are agglomerated and cell-classes/ lineages are analyzed in isolation from the remaining cell classes using SC3 consensus clustering into sub-clusters (Astrocyte sub-clusters are shown as an example). These sub-clusters are used for downstream analysis.
\end{abstract}

Additional file 2. Genes used in gene set enrichment analysis [10], in cell classifier, and discovered cell-type marker genes based on single nuclei RNAseq data

Additional file 3. Differential gene expression patterns in neurons. A) tSNE plot showing 9 different neuronal clusters. B) Here we divided up nuclei in the tSNE plot into HD (blue) and control (red). Some of the clusters appear relatively homogeneous with respect to condition, while others appear more mixed. C) Differential gene expression as a volcano plot, showing some of the highly differentially expressed genes. D) GO terms and Reactome pathway enrichment analysis of genes significantly increased in HD over all neurons. E) GO terms and Reactome pathway enrichment analysis of genes significantly decreased in HD over all neurons. The source of the GO term is color coded. P value of enrichment is represented by the length of the bar. F) Gene expression heat map of cluster markers showing nuclei (Columns) and specific genes (Rows). Condition (Con versus HD) and neuronal clusters are colorcoded on the top and bottom, respectively. Cluster-specific gene markers were identified using Wilcoxon signed rank test comparing gene ranks in the cluster with the highest mean expression against all others. p-values were adjusted using the "Holm" method. G) Examples of in situ hybridization of 4 of the neuronal genes (๔ 2010 Allen Institute for Brain Science. Allen Human Brain Atlas. Available from: human.brain-map.org). Scale bars: GOT1 $100 \mu \mathrm{m}$, others $200 \mu \mathrm{m}$.

Additional file 4. Supplementary Data. (1) Raw counts and RPKM counts of Bulk RNAseq data. (2)Results of differential gene expression analysis of each astrocytic cluster against all other clusters.

Additional file 5. Results of differential gene expression analysis of Bulk RNAseq controled for age and gender with log fold change threshold of 1.5

Additional file 6. Cortical thickness of the cingulate in HD. Representative images of cortical thickness measurements performed in sections stained for CD44 (A), Hematoxylin and Eosin (H\&E) (C), and Cresyl violet (E). Bar graphs showing average cortical thickness in individual cases in in the CD44 immunostain (B), with two regions quantified highlighted in blue and red. Bar graphs showing average cortical thickness of control and HD sections stained for H\&E (D) and 
Cresyl violet $(\mathbf{F})$. The regions quantified in the cingulate cortex are colorcoded in the images, which is reflected in the bar graphs. No significant differences were identified between control and HD using unpaired $t$ tests. $\mathrm{N}=4$ control and $5 \mathrm{HD}$ for CD44 immunostain, 6-9 HD and 6-8 control for H\&E, and 5-8 HD and 6-7 control Cresyl violet. G) Immunohistochemical staining for GFAP, Glutamine Synthetase (GS), and ALDH1L1 of a representative control and the Juvenile Huntington

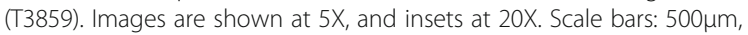
inset scale bar: $50 \mu \mathrm{m}$.

Additional file 7. Gene set variation analysis (GSVA) of the average normalized expression of all nuclei in one cell-class/type. Cell-type specific gene sets derived from the literature (A OA and JEG) and Gill et al.53 (B) are shown in the rows. Cell-types are shown in columns. The $z$ scaled enrichment scores of the cell-type averages are shown in the heat maps (A-B). The proportions of cell-types in Control (Right) and HD (Left) nuclei. Percentages per cell-type are shown in the pie chart (C). Bar-plots of count of nuclei per cell-type per case (D). Barplots of the proportions of cell-type per case ( $C=$ Control, $H=H D)(\mathbf{E})$

Additional file 8. Supplementary Movie 1. Three dimensional view of Figure $3 b$

Additional file 9. Differential gene expression between control and HD Astrocytic nuclei, GO term enriched in top differentially upregulated and downregulated genes

Additional file 10. Complement factor 3 (C3) immunostaining in the HD caudate and cingulate. A-B) Micrographs of immunostaining for C3 in the cingulate cortex (A) and caudate nucleus (B) of control and HD grade III/IV taken at 10X (100X total magnification). The boxed areas are shown at 40X in the lower panels (400X total magnification). C-D) Dual immunostaining for C3 (green) and GFAP (red -C) or LN3 (red - D) in the caudate nucleus of a representative HD case (C). Nuclei stained with DAPI are shown in blue. Scale bars indicate \#\#\#\#. A total of 3-4 cases per group were examined.

Additional file 11. Differential correlation analysis between astrocytic genes in HD and control

Additional file 12. Astrocytic gene co-expression modules, module information, module genes, GO term enrichment in modules

Additional file 13. Validation of astrocytic sub-clusters. A) Astrocytes in a control cingulate cortex. Arrows indicate astrocytes that are ALDH1L1+/MT-/ GFAP- (example of Astrocyte Clusters 3 or 4). The arrowhead indicates an astrocyte that is ALDH1L1+/MT+/GFAP-weak (example of Astrocyte Cluster 1). The two large reactive GFAP+/MT- astrocytes (not indicated by arrows) are examples of cluster 6 . A merged panel is displayed on the right. B) Astrocytes in an HD cingulate cortex. Arrows indicate astrocytes that are ALDH1L1+/MT-weak/GFAP+ (example of Astrocyte Cluster 5). Arrowheads indicate astrocytes that are ALDH1L1+/MT+/GFAP+ (example of Astrocyte Cluster 2). C) Astrocytes in an HD cingulate cortex. Arrows indicate astrocytes that are ALDH1L1+/MT+/GFAP- or weak (example of Astrocyte Cluster 1). Arrowheads indicate astrocytes that are ALDH1L1+/MT+/GFAP+ (example of Astrocyte Cluster 2). GFAP (green), ALDH1L1 (red), MT (cyan), DAPI (white). Confocal microscopy in all panels; single optical planes are shown. Scale bar $=20 \mu \mathrm{m}$

Additional file 14. Microglial gene expression alterations in the cingulate cortex. A) Differential gene expression heatmap showing a subset of significantly differentially expressed microglial genes in control and HD cingulate cortex - cingulate cortex shown in red in the top right pictogram (Microglial gene list was adapted from Patir et al [40]). B) Principle component analysis plot of microglia nuclei. Control nuclei are shown in red, HD in blue. C) Clustering of control (circles) and HD (triangles) nuclei shows they cluster separately. Colors denote clusters as determined by SC3 consensus clustering (K=2). D) Differential genes expression of between control and HD microglial nuclei displayed as a volcano plot with significance set at $p<0.05$ - genes with $-\log 10 p$ value (LogPV) of $>3$ are shown in blue, and those with - logPV of $>3$ and log2 fold change $>2$ are shown in red. Analysis was performed in EdgeR using the likelihood ratio test. E) Differential genes expression heatmap between control (red bar) and HD (blue bar) nuclei showing significantly differentially expressed genes (- $\log 10 \mathrm{p}$ value scale shown on the left). Significance was determined using Kruskal-Wallis test $(p<0.05)$.
Additional file 15. Neuronal loss and dysfunction in the HD cingulate cortex. A) Representative images of crystal violet stained cingulate cortex sections from a control case and a grade $4 \mathrm{HD}$. The subcortical white matter is shown in the lower right corner. Note the relative abundance of the small "glial" nuclei in the HD cortex. Scale bar $=100$ um. B) Representative images of cells with large nuclear area (5th quantile $>104$ um2) in the upper row and cells with small nuclear area (1st quantile < 30.8 um2) in the lower row. These areas correspond to neurons and glia, respectively. C) Quantification of the relative proportions of nuclei quantified ( $y$-axis) within different area ranges ( $x$-axis). Boxplots are shown in addition to points representing individual cases. Clue indicates $\mathrm{HD}$ and red controls. $\mathrm{N}=9$ for control $\mathrm{N}=8$ for $\mathrm{HD}$. ***: $\mathrm{p}$ value $<0.001$, $* * * * *$ : $p$ value $<0.000001$. D) GO term and Reactome pathway enrichment analysis of genes significantly downregulated in $\mathrm{HD}$ in the bulk RNAseq analysis of the cingulate cortex. All of the pathways shown are significantly enriched after Benjamini-Hochberg False discovery rate correction $(<0.05)$. The source of the GO term is color coded. P value of enrichment is represented by the length of the bar per gene ontology.

Additional file 16. Differential gene expression between control and HD neuronal nuclei, GO term enriched in top differentially upregulated and downregualted genes.

Additional file 17. Microglial genes shown in heatmap of figure S6A

\section{Acknowledgements}

We thank Sufian Al-Ahmad and Ahmad Aby-Aysheh from Al-Fardthakh for support with aspects of bulk RNAseq data analysis, Dr. Claudia Deoge for help with RNAscope, and the immunohistochemistry core in the Department of Pathology and Cell Biology at Columbia University Irving Medical Center for help with GFAP-HTT immunostains. We are grateful to the Hereditary Neurological Disease Centre, Wichita, KS, for sending tissues of their HD patients.

\section{Consent to participate}

Not applicable.

\section{Authors' contributions}

OA-D and JEG designed the study, OA-D, AAS, AS, KO, YL, IA performed the studies, JPV and JEG performed neuropatholopgical examinations of tissues, OA-D and VM did the bioinfomatics analyses, OA-D and JEG wrote the manuscript, all authors edited and approved the manuscript.

\section{Funding}

This work was funded by the Hereditary Disease Foundation, Taub Institute for the Aging Brain, and the Department of Pathology and Cell Biology at Columbia University. This research was supported by the Digital Computational Pathology Laboratory in the Department of Pathology and Cell Biology at Columbia University Irving Medical Center.

\section{Availability of data and materials}

Data for bulk RNAseq analysis is provided as a csv file with raw and normalized counts (Supplementary data). Data for snRNAseq can be queried using an interactive web app: https://vmenon.shinyapps.io/hd_sn_rnaseq/. All other data is available upon reasonable request.

\section{Ethics approval and consent to participate}

Not applicable.

\section{Competing interests}

The authors declare that they have no competing interests.

\section{Author details}

'Department of Pathology \& Cell Biology, Columbia University, New York City, NY, USA. ${ }^{2}$ Department of Neurosurgery, Columbia University, New York City, NY, USA. ${ }^{3}$ College of Medicine, Alfaisal University, Riyadh, Saudi Arabia. ${ }^{4}$ Department of Anatomy, Histology and Embryology, Semmelweiss University, Budapest, Hungary. ${ }^{5}$ Department of Neurology, Columbia University, New York City, NY, USA. 
Received: 31 December 2019 Accepted: 5 January 2020 Published online: 18 February 2020

\section{References}

1. Ament SA, Pearl JR, Cantle JP, Bragg RM, Skene PJ, Coffey SR, Bergey DE, Wheeler VC, MacDonald ME, Baliga NS et al (2018) Transcriptional regulatory networks underlying gene expression changes in Huntington's disease. Mol Syst Biol 14. https://doi.org/10.15252/msb.20167435

2. $\quad$ Arzberger T, Krampfl K, Leimgruber S, Weindl A (1997) Changes of NMDA receptor subunit (NR1, NR2B) and glutamate transporter (GLT1) mRNA expression in Huntington's disease - an in situ hybridization study. J Neuropathol Exp Neurol 56:440-454. https://doi.org/10.1097/00005072199704000-00013

3. Benraiss A, Wang S, Herrlinger S, Li X, Chandler-Militello D, Mauceri J, Burm HB, Toner M, Osipovitch M, Jim Xu Q et al (2016) Human glia can both induce and rescue aspects of disease phenotype in Huntington disease. Nat Commun 7. https://doi.org/10.1038/ncomms11758

4. Björkqvist M, Wild EJ, Thiele J, Silvestroni A, Andre R, Lahiri N, Raibon E, Lee RV, Benn CL, Soulet D et al (2008) A novel pathogenic pathway of immune activation detectable before clinical onset in Huntington's disease. J Exp Med 205:1869-1877. https://doi.org/10.1084/jem.20080178

5. Bradford J, Shin J-Y, Roberts M, Wang C-E, Li X-J, Li S (2009) Expression of mutant huntingtin in mouse brain astrocytes causes age-dependent neurological symptoms. Proc Natl Acad Sci 106:22480-22485. https://doi. org/10.1073/pnas.0911503106

6. Darmanis S, Sloan SA, Zhang Y, Enge M, Caneda C, Shuer LM, Hayden Gephart MG, Barres BA, Quake SR (2015) A survey of human brain transcriptome diversity at the single cell level. Proc Natl Acad Sci 112:72857290. https://doi.org/10.1073/pnas.1507125112

7. Diaz-Castro B, Gangwani MR, Yu X, Coppola G, Khakh BS (2019) Astrocyte molecular signatures in Huntington's disease. Sci Transl Med 11

8. Dobin A, Davis CA, Schlesinger F, Drenkow J, Zaleski C, Jha S, Batut P, Chaisson M, Gingeras TR (2012) STAR: ultrafast universal RNA-seq aligner. Bioinformatics 29:15-21. https://doi.org/10.1093/bioinformatics/bts635

9. Faideau M, Kim J, Cormier K, Gilmore R, Welch M, Auregan G, Dufour N, Guillermier M, Brouillet E, Hantraye P et al (2010) In vivo expression of polyglutamine-expanded huntingtin by mouse striatal astrocytes impairs glutamate transport: a correlation with Huntington's disease subjects. Hum Mol Genet 19:3053-3067. https://doi.org/10.1093/hmg/ddq212

10. Gill BJ, Pisapia DJ, Malone HR, Goldstein H, Lei L, Sonabend A, Yun J, Samanamud J, Sims JS, Banu M et al (2014) MRI-localized biopsies reveal subtype-specific differences in molecular and cellular composition at the margins of glioblastoma. Proc Natl Acad Sci 111:12550-12555. https://doi. org/10.1073/pnas.1405839111

11. Habib N, Avraham-Davidi I, Basu A, Burks T, Shekhar K, Hofree M, Choudhury SR, Aguet F, Gelfand E, Ardlie K et al (2017) Massively parallel single-nucleus RNA-seq with DroNc-seq. Nat Methods 14:955-958. https://doi.org/10.1038/ nmeth.4407

12. Hänzelmann S, Castelo R, Guinney J (2013) GSVA: gene set variation analysis for microarray and RNA-Seq data. BMC Bioinformatics 14:7. https://doi.org/ 10.1186/1471-2105-14-7

13. Hedreen JC, Peyser CE, Folstein SE, Ross CA (1991) Neuronal loss in layers V and $\mathrm{VI}$ of cerebral cortex in Huntington's disease. Neurosci Lett 133:257261. https://doi.org/10.1016/0304-3940(91)90583-f

14. Hensman Moss DJ, Flower MD, Lo KK, Miller JRC, van Ommen G-JB, 't Hoen PAC, Stone TC, Guinee A, Langbehn DR, Jones L et al (2017) Huntington's disease blood and brain show a common gene expression pattern and share an immune signature with Alzheimer's disease. Sci Rep 7. https://doi. org/10.1038/srep44849

15. Hodges A, Strand AD, Aragaki AK, Kuhn A, Sengstag T, Hughes G, Elliston LA, Hartog C, Goldstein DR, Thu D et al (2006) Regional and cellular gene expression changes in human Huntington's disease brain. Hum Mol Genet 15:965-977. https://doi.org/10.1093/hmg/ddl013

16. Hsiao H-Y, Chen Y-C, Chen H-M, Tu P-H, Chern Y (2013) A critical role of astrocyte-mediated nuclear factor-KB-dependent inflammation in Huntington's disease. Hum Mol Genet 22:1826-1842. https://doi.org/10. 1093/hmg/ddt036

17. Hu P, Fabyanic E, Kwon DY, Tang S, Zhou Z, Wu H (2017) Dissecting celltype composition and activity-dependent transcriptional state in mammalian brains by massively parallel single-nucleus RNA-Seq. Mol Cell 68:1006-1015.e1007. https://doi.org/10.1016/j.molcel.2017.11.017
18. Jäkel S, Agirre E, Mendanha Falcão A, van Bruggen D, Lee KW, Knuesel I, Malhotra D, Ffrench-Constant C, Williams A, Castelo-Branco G (2019) Altered human oligodendrocyte heterogeneity in multiple sclerosis. Nature 566 : 543-547. https://doi.org/10.1038/s41586-019-0903-2

19. Kiselev VY, Kirschner K, Schaub MT, Andrews T, Yiu A, Chandra T, Natarajan KN, Reik W, Barahona M, Green AR et al (2017) SC3: consensus clustering of single-cell RNA-seq data. Nat Methods 14:483-486. https://doi.org/10.1038/ nmeth.4236

20. Kolde R, Vilo J (2015) GOsummaries: an R package for visual functional annotation of experimental data. F1000Res 4:574. https://doi.org/10.12688/ f1000research.6925.1

21. Kopylova E, Noé L, Touzet H (2012) SortMeRNA: fast and accurate filtering of ribosomal RNAs in metatranscriptomic data. Bioinformatics 28:3211-3217. https://doi.org/10.1093/bioinformatics/bts611

22. Krishnaswami SR, Grindberg RV, Novotny M, Venepally P, Lacar B, Bhutani K, Linker SB, Pham S, Erwin JA, Miller JA et al (2016) Using single nuclei for RNA-seq to capture the transcriptome of postmortem neurons. Nat Protoc 11:499-524. https://doi.org/10.1038/nprot.2016.015

23. Labadorf A, Hoss AG, Lagomarsino V, Latourelle JC, Hadzi TC, Bregu J, MacDonald ME, Gusella JF, Chen J-F, Akbarian S et al (2015) RNA sequence analysis of human Huntington disease brain reveals an extensive increase in inflammatory and developmental gene expression. PLoS One 10:e0143563. https://doi.org/10.1371/journal.pone.0143563

24. Lake BB, Ai R, Kaeser GE, Salathia NS, Yung YC, Liu R, Wildberg A, Gao D, Fung HL, Chen $S$ et al (2016) Neuronal subtypes and diversity revealed by single-nucleus RNA sequencing of the human brain. Science 352:1586-1590. https://doi.org/10.1126/science.aaf1204

25. Lake BB, Codeluppi S, Yung YC, Gao D, Chun J, Kharchenko PV, Linnarsson S, Zhang K (2017) A comparative strategy for single-nucleus and single-cell transcriptomes confirms accuracy in predicted cell-type expression from nuclear RNA. Sci Rep 7. https://doi.org/10.1038/s41598017-04426-w

26. Lê S, Josse J, Husson F (2008) FactoMineR: AnRPackage for multivariate analysis. J Stat Softw 25. https://doi.org/10.18637/jss.v025.i01

27. Liddelow SA, Guttenplan KA, Clarke LE, Bennett FC, Bohlen CJ, Schirmer L, Bennett ML, Münch AE, Chung W-S, Peterson TC et al (2017) Neurotoxic reactive astrocytes are induced by activated microglia. Nature 541:481-487. https://doi.org/10.1038/nature21029

28. Lien ES (2007) Genome-wide expression of genome-wide atlas of gene expression in the adult mouse brain. Nature 445:168-176

29. Lin L, Park JW, Ramachandran S, Zhang Y, Tseng Y-T, Shen S, Waldvogel HJ, Curtis MA, Faull RLM, Troncoso JC et al (2016) Transcriptome sequencing reveals aberrant alternative splicing in Huntington's disease. Hum Mol Genet 25:3454-3466. https://doi.org/10.1093/hmg/ddw187

30. Love MI, Huber W, Anders S (2014) Moderated estimation of fold change and dispersion for RNA-seq data with DESeq2. Genome biology 15. https:// doi.org/10.1186/s13059-014-0550-8

31. Mathys H, Davila-Velderrain J, Peng Z, Gao F, Mohammadi S, Young JZ, Menon M, He L, Abdurrob F, Jiang X et al (2019) Single-cell transcriptomic analysis of Alzheimer's disease. Nature 570:332-337. https://doi.org/10.1038/ s41586-019-1195-2

32. McCarthy DJ, Campbell KR, Lun ATL, Wills QF (2017) Scater: preprocessing, quality control, normalization and visualization of single-cell RNA-seq data in R. bioinformatics: btw777. https://doi.org/10.1093/ bioinformatics/btw777

33. McCarthy DJ, Chen Y, Smyth GK (2012) Differential expression analysis of multifactor RNA-Seq experiments with respect to biological variation. Nucleic Acids Res 40:4288-4297. https://doi.org/10.1093/nar/gks042

34. McColgan P, Tabrizi SJ (2017) Huntington's disease: a clinical review. Eur J Neurol 25:24-34. https://doi.org/10.1111/ene.13413

35. McKenzie AT, Katsyv I, Song W-M, Wang M, Zhang B (2016) DGCA: a comprehensive $\mathrm{R}$ package for differential gene correlation analysis. BMC Syst Biol 10. https://doi.org/10.1186/s12918-016-0349-1

36. McKenzie AT, Wang M, Hauberg ME, Fullard JF, Kozlenkov A, Keenan A, Hurd YL, Dracheva S, Casaccia P, Roussos P et al (2018) Brain cell type specific gene expression and co-expression network architectures. Sci Rep 8. https://doi.org/10.1038/s41598-018-27293-5

37. Mehrabi NF, Waldvogel HJ, Tippett LJ, Hogg VM, Synek BJ, Faull RLM (2016) Symptom heterogeneity in Huntington's disease correlates with neuronal degeneration in the cerebral cortex. Neurobiol Dis 96:67-74. https://doi.org/ 10.1016/j.nbd.2016.08.015 
38. Mina E, van Roon-Mom W, Hettne K, van Zwet E, Goeman J, Neri C, A.C. 't Hoen P, Mons B, Roos M (2016) Common disease signatures from gene expression analysis in Huntington's disease human blood and brain. Orphan J Rare Dis 11. https://doi.org/10.1186/s13023-016-0475-2

39. Olah M, Patrick E, Villani A-C, Xu J, White CC, Ryan KJ, Piehowski P, Kapasi A, Nejad P, Cimpean M. et al (2018) A transcriptomic atlas of aged human microglia. Nat Commun. 9: Doi https://doi.org/10.1038/s41467-018-02926-5

40. Penkowa M, Carrasco J, Giralt M, Moos T, Hidalgo J (1999) CNS wound healing is severely depressed in Metallothionein I- and II-deficient mice. J Neurosci 19:2535-2545. https://doi.org/10.1523/jneurosci.19-07-02535.1999

41. Petukhov V, Guo J, Baryawno N, Severe N, Scadden DT, Samsonova MG, Kharchenko PV (2018) dropEst: pipeline for accurate estimation of molecular counts in droplet-based single-cell RNA-seq experiments. Genome Biol 19: 78. https://doi.org/10.1186/s13059-018-1449-6

42. Pollen Alex A, Nowakowski Tomasz J, Chen J, Retallack H, Sandoval-Espinosa C, Nicholas Cory R, Shuga J, Liu Siyuan J, Oldham Michael C, Diaz A et al (2015) Molecular identity of human outer radial glia during cortical development. Cell 163:55-67. https://doi.org/10.1016/j.cell.2015.09.004

43. Robinson MD, McCarthy DJ, Smyth GK (2009) edgeR: a bioconductor package for differential expression analysis of digital gene expression data. Bioinformatics 26:139-140. https://doi.org/10.1093/bioinformatics/btp616

44. Rüb U, Seidel K, Heinsen H, Vonsattel JP, den Dunnen WF, Korf HW (2016) Huntington's disease (HD): the neuropathology of a multisystem neurodegenerative disorder of the human brain. Brain Pathol 26:726-740. https://doi.org/10.1111/bpa.12426

45. Rüb U, Vonsattel JPG, Heinsen H, Korf H-W (2015) The neuropathology of Huntington's disease: classical findings, recent developments and correlation to functional Neuroanatomy. Advances in anatomy, embryology and cell biology. 217:1-146.

46. Santos CRA, Martinho A, Quintela T, Gonçalves I (2011) Neuroprotective and neuroregenerative properties of metallothioneins. IUBMB Life 64:126-135. https://doi.org/10.1002/iub.585

47. Sekar S, McDonald J, Cuyugan L, Aldrich J, Kurdoglu A, Adkins J, Serrano G, Beach TG, Craig DW, Valla J et al (2015) Alzheimer's disease is associated with altered expression of genes involved in immune response and mitochondrial processes in astrocytes. Neurobiol Aging 36:583-591. https:// doi.org/10.1016/j.neurobiolaging.2014.09.027

48. Selkoe DJ, Salasar FJ, Abraham C, Kosik KS (1982) Huntington's disease: changes in striatal proteins reflect astrocytic gliosis. Brain Res 245:117-125. https://doi.org/10.1016/0006-8993(82)90344-4

49. Song W-M, Zhang B (2015) Multiscale embedded gene co-expression network analysis. PLoS Comput Biol 11:e1004574. https://doi.org/10.1371/ journal.pcbi.1004574

50. Sorolla MA, Reverter-Branchat G, Tamarit J, Ferrer I, Ros J, Cabiscol E (2008) Proteomic and oxidative stress analysis in human brain samples of Huntington disease. Free Radic Biol Med 45:667-678. https://doi.org/10. 1016/j.freeradbiomed.2008.05.014

51. Sosunov AA, Wu X, Tsankova NM, Guilfoyle E, McKhann GM, Goldman JE (2014) Phenotypic heterogeneity and plasticity of Isocortical and hippocampal astrocytes in the human brain. J Neurosci 34:2285-2298. https://doi.org/10.1523/jneurosci.4037-13.2014

52. Tereshchenko A, Magnotta V, Epping E, Mathews K, Espe-Pfeifer P, Martin E, Dawson J, Duan W, Nopoulos P (2019) Brain structure in juvenile-onset Huntington disease. Neurology 92:e1939-e1947. https://doi.org/10.1212/wnl. 0000000000007355

53. Tong $X, A o ~ Y$, Faas GC, Nwaobi SE, Xu J, Haustein MD, Anderson MA, Mody I, Olsen ML, Sofroniew MV et al (2014) Astrocyte Kir4.1 ion channel deficits contribute to neuronal dysfunction in Huntington's disease model mice. Nat Neurosci 17:694-703. https://doi.org/10.1038/nn.3691

54. Trapnell C, Cacchiarelli D, Grimsby J, Pokharel P, Li S, Morse M, Lennon NJ, Livak KJ, Mikkelsen TS, Rinn JL (2014) The dynamics and regulators of cell fate decisions are revealed by pseudotemporal ordering of single cells. Nat Biotechnol 32:381-386. https://doi.org/10.1038/nbt.2859

55. Tsai HH, Li H, Fuentealba LC, Molofsky AV, Taveira-Marques R, Zhuang H, Tenney A, Murnen AT, Fancy SPJ, Merkle F et al (2012) Regional astrocyte allocation regulates CNS synaptogenesis and repair. Science 337:358-362. https://doi.org/10.1126/science.1222381

56. van Lookeren CM, Thibodeaux H, van Bruggen N, Cairns B, Gerlai R, Palmer JT, Williams SP, Lowe DG (1999) Evidence for a protective role of metallothionein-1 in focal cerebral ischemia. Proc Natl Acad Sci 96:1287012875. https://doi.org/10.1073/pnas.96.22.12870
57. Vonsattel J-P, Myers RH, Stevens TJ, Ferrante RJ, Bird ED, Richardson EP (1985) Neuropathological classification of Huntington's disease. J Neuropathol Exp Neurol 44:559-577. https://doi.org/10.1097/00005072198511000-00003

58. Wakai M, Takahashi A, Hashizume Y (1993) A histometrical study on the globus pallidus in Huntington's disease. J Neurol Sci 119:18-27. https://doi. org/10.1016/0022-510x(93)90187-4

59. Wakida K, Shimazawa M, Hozumi I, Satoh M, Nagase H, Inuzuka T, Hara H (2007) Neuroprotective effect of erythropoietin, and role of metallothionein1 and -2, in permanent focal cerebral ischemia. Neuroscience 148:105-114. https://doi.org/10.1016/j.neuroscience.2007.04.063

60. Warnes GR, Bolker B, Bonebakker L, Gentleman R, Huber W, Liaw A, Lumley T, Maechler M, Magnusson A, Moeller S (2009) Gplots: various R programming tools for plotting data. $\mathrm{R}$ package version 2: 1

61. Wilkinson L (2011) ggplot2: elegant graphics for data analysis by WICKHAM, H. Biometrics 67:678-679. https://doi.org/10.1111/j.1541-0420.2011.01616.X

62. Wu L, Li J, Liu T, Li S, Feng J, Yu Q, Zhang J, Chen J, Zhou Y, Ji J et al (2019) Quercetin shows anti-tumor effect in hepatocellular carcinoma LM3 cells by abrogating JAK2/STAT3 signaling pathway. Cancer Med 8:4806-4820. https://doi.org/10.1002/cam4.2388

63. Zamanian JL, Xu L, Foo LC, Nouri N, Zhou L, Giffard RG, Barres BA (2012) Genomic analysis of reactive Astrogliosis. J Neurosci 32:6391-6410. https:// doi.org/10.1523/jneurosci.6221-11.2012

\section{Publisher's Note}

Springer Nature remains neutral with regard to jurisdictional claims in published maps and institutional affiliations.
Ready to submit your research? Choose BMC and benefit from:

- fast, convenient online submission

- thorough peer review by experienced researchers in your field

- rapid publication on acceptance

- support for research data, including large and complex data types

- gold Open Access which fosters wider collaboration and increased citations

- maximum visibility for your research: over $100 \mathrm{M}$ website views per year

At BMC, research is always in progress.

Learn more biomedcentral.com/submissions 